Article

\title{
Triterpene-Based Carboxamides Act as Good Inhibitors of Butyrylcholinesterase
}

\author{
Anne Loesche ${ }^{1}{ }^{(}$, Michael Kahnt ${ }^{1}\left(\mathbb{D}\right.$, Immo Serbian ${ }^{1}\left(\mathbb{D}\right.$, Wolfgang Brandt ${ }^{2}$ and René Csuk ${ }^{1, *(1)}$ \\ 1 Martin-Luther-University Halle-Wittenberg, Organic Chemistry, Kurt-Mothes-Str. 2, \\ D-06120 Halle (Saale), Germany; \\ anne.loesche@chemie.uni-halle.de (A.L.); michael.kahnt@chemie.uni-halle.de (M.K.); \\ immo.serbian@chemie.uni-halle.de (I.S.) \\ 2 Leibniz Institute of Plant Biochemistry, Bioorganic Chemistry, Weinberg 3, D-06120 Halle (Saale), Germany; \\ wbrandt@ipb-halle.de \\ * Correspondence: rene.csuk@chemie.uni-halle.de; Tel.: +49-345-55-25660
}

Academic Editors: Eva E. Rufino-Palomares and José Antonio Lupiáñez

Received: 12 February 2019; Accepted: 2 March 2019; Published: 7 March 2019

check for updates

\begin{abstract}
A set of overall 40 carboxamides was prepared from five different natural occurring triterpenoids including oleanolic, ursolic, maslinic, betulinic, and platanic acid. All of which were derived from ethylene diamine holding an additional substituent connected to the ethylene diamine group. These derivatives were evaluated regarding their inhibitory activity of the enzymes acetylcholinesterase (AChE) and butyrylcholinesterase (BChE) employing Ellman's assay. We further determined the type of inhibition and inhibition constants. Carboxamides derived from platanic acid have been shown to be potent and selective BChE inhibitors. Especially the mixed-type inhibitor (3ß)-N-(2-pyrrolidin-1-ylethyl)-3-acetyloxy-20-oxo-30-norlupan-28-amide (35) showed a remarkably low $\mathrm{K}_{\mathrm{i}}$ of $0.07 \pm 0.01 \mu \mathrm{M}\left(\mathrm{K}_{\mathrm{i}}{ }^{\prime}=2.38 \pm 0.48 \mu \mathrm{M}\right)$ for the inhibition of BChE.
\end{abstract}

Keywords: acetylcholinesterase; butyrylcholinesterase; triterpenoids

\section{Introduction}

It is now more than a century since Alois Alzheimer, a German physician, described a new disease of the brain [1], being today one of the most threatening plagues for the elderly and one of the greatest challenges for chemists and biologists. The eponymous disease is today one of the greatest scourges of humanity. Alzheimer's disease (AD), the most common cause of dementia, is a neurodegenerative disorder, causing the progressive loss of cognitive functions and memory. As a result of demographic changes, the number of $\mathrm{AD}$ patients is steadily rising. This disease is characterized by an increasing decline in acetylcholine ( $\mathrm{ACh}$, neurotransmitter) levels in the cholinergic system [2]. A common strategy for the management of AD is to develop inhibitors that suppress the degradation of ACh caused by hydrolases acetylcholinesterase (AChE, EC 3.1.1.7) and butyrylcholinesterase (BChE, EC 3.1.1.8).

Ursolic acid (UA), oleanolic acid (OA), maslinic acid (MA), betulinic acid (BA), and platanic acid (PA) are naturally occurring pentacyclic triterpenoids (Figure 1), which are widely distributed in various plants. Triterpenes and their derivatives represent a group of pharmacologically interesting substances showing a variety of biological activities such as antitumor [3], antiviral, and anti-HIV [4,5], antibacterial [6,7], and antifungal [8] properties. Pentacyclic triterpenoic acids have already been tested for cholinergic activities with moderate anti-cholinesterase activity $[9,10]$. Cyclic terpene derivatives, which act as inhibitors of cholinesterases, have been our research focus for a long time. Results from our group have demonstrated that structural modifications of the terpenoid backbone have a high impact onto the inhibitory potential for cholinesterases (ChEs) [11-13]. Exceptionally good ChE 
inhibition has been found for some amino derivatives of platanic acid [12], as well as for amides of dehydroabietylamine [14] and 12-hydroxydehydroabietylamine [15].

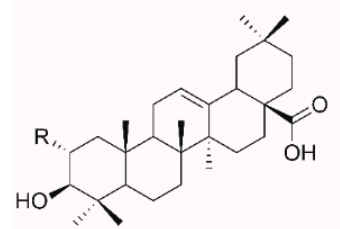

$2 \mathrm{R}=\mathrm{H} \quad(\mathrm{OA})$ $3 \mathrm{R}=\mathrm{OH}(\mathrm{MA})$

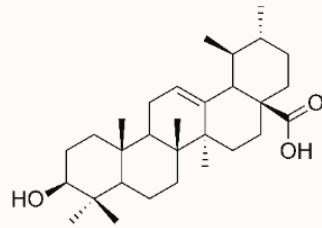

1 (UA)

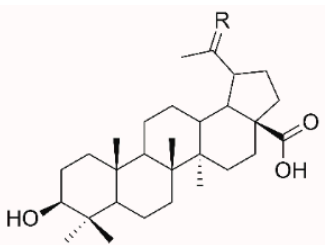

$4 \mathrm{R}=\mathrm{CH}_{2}(\mathrm{BA})$

Figure 1. Structures of triterpenoic acids 1-5.

In patients, $\mathrm{BChE}$ activity increases with progression of $\mathrm{AD}$ while the level of $\mathrm{AChE}$ remains constant [16]. Therefore, selective BChE inhibitors represent legitimate therapeutic options to improve the deficit in the neurotransmitter ACh. Deduced from the results of our previous studies, UA, OA, MA, BA and PA carboxamides holding a terminal amino moiety were synthesized from the parent pentacyclic triterpenoic acids. Altogether, 40 different compounds were synthesized and screened for their ChE inhibitory activity using Ellman's assay.

\section{Results and Discussion}

\subsection{Chemistry}

Acetylation of triterpenoic acids (Figure 1, 1-5) furnished acetates 6-10 (Scheme 1). Reaction of 6-10 with oxalyl chloride and subsequent treatment with various amines derived from ethylene diamine gave carboxamides 11-15, 21-25, 31-35 and 41-45. Their deacetylation with $\mathrm{KOH} / \mathrm{MeOH}$ gave compounds $16-20,26-30,36-40$ and $46-50$, respectively.

\begin{tabular}{|c|c|c|c|c|c|c|c|c|c|}
\hline & & & & & & & & & \\
\hline & $\mathrm{R}^{2}$ & & & & & & & & \\
\hline & $\mathrm{R}^{1}$ & $A c$ & $\mathrm{H}$ & Ac & $\mathrm{H}$ & $A c$ & $\mathrm{H}$ & Ac & $\mathrm{H}$ \\
\hline \multirow{5}{*}{ 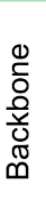 } & UA & 11 & 16 & 21 & 26 & 31 & 36 & 41 & 46 \\
\hline & $O A$ & 12 & 17 & 22 & 27 & 32 & 37 & 42 & 47 \\
\hline & MA & 13 & 18 & 23 & 28 & 33 & 38 & 43 & 48 \\
\hline & BA & 14 & 19 & 24 & 29 & 34 & 39 & 44 & 49 \\
\hline & PA & 15 & 20 & 25 & 30 & 35 & 40 & 45 & 50 \\
\hline
\end{tabular}

Scheme 1. Generalized representation of 1-5 and preparation of triterpenoic carboxamides 11-50: (a) $\mathrm{Ac}_{2} \mathrm{O}, \mathrm{DCM}$, TEA, $25^{\circ} \mathrm{C}, 2$ days, 90-96\%; (b) oxalyl chloride, DCM, DMF, 0-25 ${ }^{\circ} \mathrm{C}, 1 \mathrm{~h}$, then amine, $25^{\circ} \mathrm{C}, 2 \mathrm{~h} ;(\mathbf{c}) \mathrm{MeOH} / \mathrm{KOH}, 25^{\circ} \mathrm{C}, 2-3$ days.

\subsection{Biology}

In this study, carboxamides with different triterpenoic backbones (ursolic, oleanolic, maslinic, betulinic, and platanic acids), and various amine residues (Scheme 1), were investigated. All compounds 11-50 (except those being not soluble under the conditions of the assay) were subjected to Ellman's assays to determine their inhibition rates and constants $\left(\mathrm{K}_{\mathrm{i}}\right.$ for competitive inhibition and $\mathrm{K}_{\mathrm{i}}{ }^{\prime}$ for uncompetitive inhibition) for the cholinesterases $\mathrm{AChE}$ and BChE. Galantamine hydrobromide (GH), one of the gold standard drugs for treating AD symptoms, was used for comparison. In general, 
pre-screening the 37 triterpenoic acid amides using $\mathrm{AChE}$ (electric eel) and $\mathrm{BChE}$ (equine serum) identified seventeen compounds as potential inhibitors with inhibition rates equal or even better than GH. The results are summarized in Figures 2 and 3 (details are found in the Supplementary Part, Table S1).

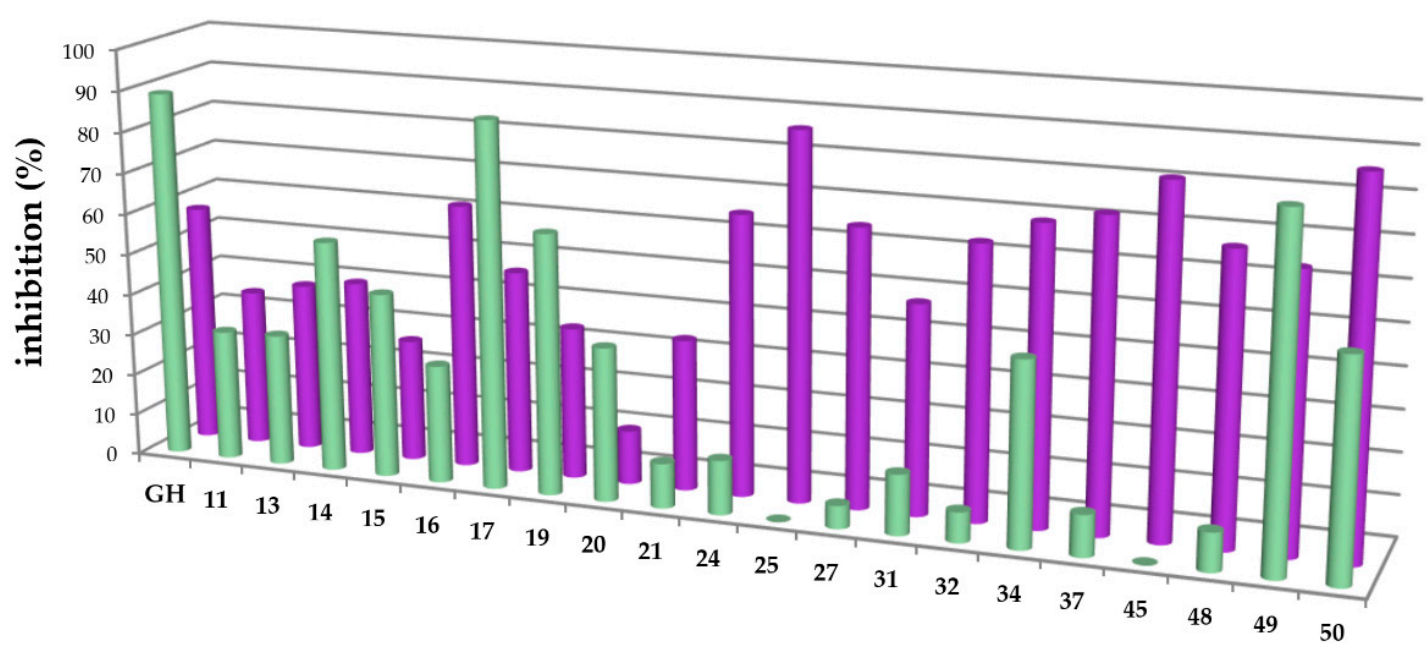

Figure 2. Percentage of inhibition of selected carboxamides and galantamine hydrobromide (GH as standard), final concentration of the inhibitor $10 \mu \mathrm{M}$, determined by Ellman's assay using acetylcholinesterase (green in the front) and butyrylcholinesterase (purple in the back).

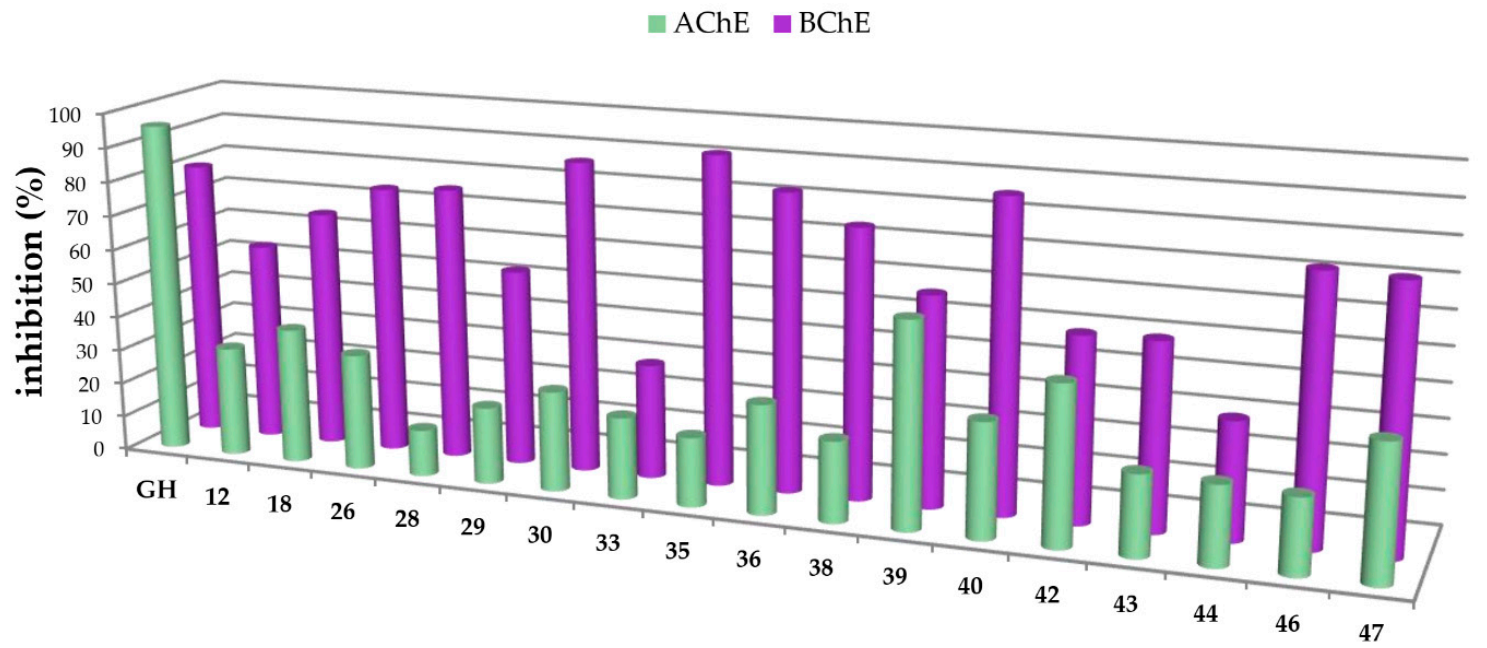

Figure 3. Percentage of inhibition of selected carboxamides and galantamine hydrobromide (GH as standard), final concentration of the inhibitor $30 \mu \mathrm{M}$, determined by Ellman's assay using acetylcholinesterase (green in the front) and butyrylcholinesterase (purple in the back).

The carboxamides showed a considerably higher inhibition for BChE than for AChE. With the exception of $17(88.61 \pm 0.22 \%)$ and 49 (82.72 $\pm 0.09 \%)$, none of the tested compounds showed any notable activity for AChE. Further kinetic studies (see Supplementary Part, Table S2) showed (3ß)-N-(2-piperidin-1-ylethyl)-3-hydroxy-lup-20(29)-en-28-amide (49) as a good mixed-type AChE inhibitor with inhibition constants: $\mathrm{K}_{\mathrm{i}}=1.00 \pm 0.09 \mu \mathrm{M}$ and $\mathrm{K}_{\mathrm{i}}{ }^{\prime}=1.42 \pm 0.08 \mu \mathrm{M}$. The results of the screening experiments suggest that many of the synthesized carboxylic acid amides inhibit $\mathrm{BChE}$ activity to a high degree. Fifteen derivatives showed promising inhibitory rates, five of which seemed to inhibit BChE almost completely (88.37-94.60\% inhibition rate). The evaluation of the 
Dixon [17], Cornish-Bowden [18], and Lineweaver-Burk [19] plots showed that all active compounds were mixed-type inhibitors with a dominating competitive part. The results from these measurements are compiled in Tables 1 and 2.

Table 1. Significant results of the BChE inhibition assay. Inhibitory constants $\left(K_{i}\right.$ and $K_{i}{ }^{\prime}$ in $\left.\mu M\right)$, determined using Ellman's assays employing butyrylcholinesterase (BChE, equine serum) with galantamine hydrobromide $(\mathbf{G H})$ as standard.

\begin{tabular}{|c|c|c|c|}
\hline Compound & $\begin{array}{l}K_{i} \text { in } \mu M / K_{i}^{\prime} \text { in } \mu M \\
\text { (Type of Inhibition) }\end{array}$ & Compound & $\begin{array}{l}K_{i} \text { in } \mu M / K_{i}{ }^{\prime} \text { in } \mu M \\
\text { (Type of Inhibition) }\end{array}$ \\
\hline GH & $\begin{array}{c}2.30 \pm 0.17 \\
\text { (competitive) }\end{array}$ & 32 & $\begin{array}{c}2.02 \pm 0.56 / 29.88 \pm 1.13 \\
\text { (mixed-type) }\end{array}$ \\
\hline 16 & $\begin{array}{c}5.55 \pm 0.12 / 12.15 \pm 0.45 \\
\text { (mixed-type) }\end{array}$ & 34 & $\begin{array}{c}0.39 \pm 0.04 />5 \\
\text { (mixed-type) }\end{array}$ \\
\hline 17 & $\begin{array}{c}1.93 \pm 0.13 / 7.68 \pm 0.61 \\
\text { (mixed-type) }\end{array}$ & 36 & $\begin{array}{c}0.47 \pm 0.08 / 2.23 \pm 0.12 \\
\text { (mixed-type) }\end{array}$ \\
\hline 21 & $\begin{array}{c}6.48 \pm 0.10 / 143.36 \pm \\
0.02 \\
\text { (mixed-type) }\end{array}$ & 37 & $\begin{array}{l}0.55 \pm 0.02 />50 \\
\text { (mixed-type) }\end{array}$ \\
\hline 24 & $\begin{array}{c}1.93 \pm 0.13 / 7.68 \pm 0.61 \\
\text { (mixed-type) }\end{array}$ & 38 & $\begin{array}{c}2.06 \pm 0.03 / 14.15 \pm 2.42 \\
\text { (mixed-type) }\end{array}$ \\
\hline 26 & $\begin{array}{c}1.11 \pm 0.09 / 3.10 \pm 0.30 \\
\text { (mixed-type) }\end{array}$ & 39 & $\begin{array}{c}3.24 \pm 0.58 />9 \\
\text { (mixed-type) }\end{array}$ \\
\hline 27 & $\begin{array}{c}1.31 \pm 0.01 / 9.07 \pm 0.02 \\
\text { (mixed-type) }\end{array}$ & 46 & $\begin{array}{c}2.37 \pm 0.02 / 7.64 \pm 0.22 \\
\text { (mixed-type) }\end{array}$ \\
\hline 28 & $\begin{array}{c}2.20 \pm 0.25 / 27.26 \pm 3.47 \\
\text { (mixed-type) }\end{array}$ & 47 & $\begin{array}{c}1.57 \pm 0.01 / 10.42 \pm 1.36 \\
\text { (mixed-type) }\end{array}$ \\
\hline 29 & $\begin{array}{c}3.52 \pm 0.07 / 16.11 \pm 0.18 \\
\text { (mixed-type) }\end{array}$ & 48 & $\begin{array}{c}2.26 \pm 0.05 / 6.21 \pm 0.47 \\
\text { (mixed-type) }\end{array}$ \\
\hline 31 & $\begin{array}{c}4.42 \pm 0.34 / 6.00 \pm 0.47 \\
\text { (mixed-type) }\end{array}$ & 49 & $\begin{array}{l}2.52 \pm 0.29 />8 \\
\text { (mixed-type) }\end{array}$ \\
\hline
\end{tabular}

Table 2. Results of the BChE inhibition assay for platanic acid derivatives. Inhibitory constants $\left(\mathrm{K}_{\mathrm{i}}\right.$ and $\mathrm{K}_{\mathrm{i}}{ }^{\prime}$ in $\mu \mathrm{M}$ ), determined using Ellman's assay employing butyrylcholinesterase (BChE, equine serum) with galantamine hydrobromide $\left(\mathbf{G H}, \mathrm{K}_{\mathrm{i}}=2.30 \pm 0.17\right)$ as standard.

\begin{tabular}{|c|c|c|c|}
\hline Compound & $\begin{array}{l}\mathrm{K}_{\mathrm{i}} \text { in } \mu \mathrm{M} / \mathrm{K}_{\mathrm{i}}{ }^{\prime} \text { in } \mu \mathrm{M} \\
\text { (Type of Inhibition) }\end{array}$ & Compound & $\begin{array}{l}\mathrm{K}_{\mathrm{i}} \text { in } \mu \mathrm{M} / \mathrm{K}_{\mathrm{i}}^{\prime} \text { in } \mu \mathrm{M} \\
\text { (Type of Inhibition) }\end{array}$ \\
\hline 15 & $\begin{array}{c}11.94 \pm 1.17 / 17.14 \pm 1.67 \\
\text { (mixed-type) }\end{array}$ & 35 & $\begin{array}{c}0.07 \pm 0.01 / 2.38 \pm 0.48 \\
\text { (mixed-type) }\end{array}$ \\
\hline 20 & $\begin{array}{c}8.37 \pm 0.67 />110 \\
\text { (mixed-type) }\end{array}$ & 40 & $\begin{array}{c}0.45 \pm 0.01 />10 \\
\text { (mixed-type) }\end{array}$ \\
\hline 25 & $\begin{array}{c}1.60 \pm 0.13 / 1.60 \pm 0.11 \\
\text { (mixed-type) }\end{array}$ & 45 & $\begin{array}{c}0.71 \pm 0.04 / 4.88 \pm 0.21 \\
\text { (mixed-type) }\end{array}$ \\
\hline 30 & $\begin{array}{c}1.12 \pm 0.01 / 4.46 \pm 0.01 \\
\text { (mixed-type) }\end{array}$ & 50 & $\begin{array}{c}0.97 \pm 0.04 / 3.09 \pm 0.03 \\
\text { (mixed-type) }\end{array}$ \\
\hline
\end{tabular}

The first set of compounds holding an aminoethyl residue, 11-14 and 16-19, were moderate inhibitors of BChE, with oleanolic acid derivative $\mathbf{1 7}$ as the only noteworthy compound of this series $\left(\mathrm{Ki}=1.93 \pm 0.13 \mu \mathrm{M}\right.$ and $\left.\mathrm{K}_{\mathrm{i}}{ }^{\prime}=7.68 \pm 0.61 \mu \mathrm{M}\right)$. The next set consisting of triterpenoic amides with a dimethylaminoethyl substituent (21-24 and 26-29) showed good inhibition with $\mathrm{K}_{\mathrm{i}}$ values between 1.1 and $6.5 \mu \mathrm{M}$, respectively. For 2-piperidin-1-ylethyl substituted derivatives (41-44 and 46-49) inhibition constants in the same range were obtained. The group of 2-pyrrolidin-1-ylethyl substituted compounds (31-34 and 36-39) delivered the best results: derivatives 34 (from betulinic acid), 36 (ursolic acid backbone) and 37 (from oleanolic acid), held excellent $\mathrm{K}_{\mathrm{i}}$ values of $0.39 \pm 0.04 \mu \mathrm{M}, 0.47 \pm 0.08 \mu \mathrm{M}$, and $0.55 \pm 0.02 \mu \mathrm{M}$, respectively. Compounds showing the highest selectivity (F, expressed by percent 
inhibition for $\mathrm{AChE}$ divided by percent inhibition for BChE) towards BChE were compounds 27 $(\mathrm{F}=0.08)$ and $32(\mathrm{~F}=0.11)$.

Because of the known inhibitory effect [12], a set of eight platanic acid derivatives was investigated (Table 2). Compounds with an aminoethyl residue (15 and 20) were the weakest BChE inhibitors in our test. Compared to the standard GH $\left(\mathrm{K}_{\mathrm{i}}=2.30 \pm 0.17 \mu \mathrm{M}\right), 2$-(dimethylamino)ethyl substituted derivatives showed good inhibition constants $\left(K_{i}=1.60 \pm 0.13 \mu \mathrm{M}\right.$ for 25 and $K_{i}=1.12 \pm 0.01 \mu \mathrm{M}$ for 30).

Compounds, carrying a piperidinyl group, showed inhibition even in nanomolar concentrations. For compound $45\left(K_{i}=0.71 \pm 0.04 \mu \mathrm{M}\right)$ and $50\left(K_{i}=0.97 \pm 0.04 \mu \mathrm{M}\right)$ excellent values were observed. As already described above, introducing a pyrrolidinyl moiety resulted in compounds with excellent $\mathrm{K}_{\mathrm{i}}$ values. A very low inhibition constant $\left(\mathrm{K}_{\mathrm{i}}=0.45 \pm 0.01 \mu \mathrm{M}\right)$ was measured for compound 40 . The most active compound was (3ß)-N-(2-pyrrolidin-1-ylethyl)-3-acetyloxy-20-oxo-30-norlupan-28-amide (35) showing inhibition constants of $\mathrm{K}_{\mathrm{i}}=0.07 \pm 0.01 \mu \mathrm{M}$ and $\mathrm{K}_{\mathrm{i}}{ }^{\prime}=2.38 \pm 0.48 \mu \mathrm{M}$ for BChE. This compound proved to be a selective $(\mathrm{F} \approx 0.21)$ inhibitor for $\mathrm{BChE}$. Except for $\mathbf{1 5}$ and 20, all PA-derived compounds showed reasonably high $\mathrm{BChE}$ selectivity.

To explain the results from the biological testing, some molecular modeling studies were performed. Table 3 summarizes the results for the most favored docking position of each ligand. From these results, nice correlations between the gold fitness values (the higher the value the better the predicted affinity between ligand enzyme), as well as the interaction energies, with the experimental $\mathrm{K}_{\mathrm{i}}$ values were established.

Table 3. Results of the docking studies with gold fitness values (PLP: Piecewise Linear Potential) and interaction energies (IE).

\begin{tabular}{cccc}
\hline Compound & $\begin{array}{c}\text { Gold Fitness } \\
\text { (ChemPLP) }\end{array}$ & $\begin{array}{c}\text { IE } \\
\text { (kcal/mol) }\end{array}$ & $\mathbf{K}_{\mathbf{i}}$ \\
\hline $\mathbf{3 1}$ & 90.0 & -57.8 & 4.42 \\
$\mathbf{3 4}$ & 98.7 & -60.4 & 0.39 \\
$\mathbf{3 5}$ & 99.0 & -64.0 & 0.07 \\
\hline
\end{tabular}

Figure 4 displays details of the docking arrangements of ligands with BChE. All ligands fit into the binding pocket-preferentially based on hydrophobic interactions (Figure 5) with the side chains of residues W79, W228, L283, Y329, and F326. The highest affinity was observed for 35 (Figure 4a). Thus a hydrogen bond of the acetyl group of $\mathbf{3 5}$ with the phenolic hydroxyl group of Y79 stabilizes the interaction with BChE. This hydrogen bond, however, is not present in compounds 31 and 34. The modifications in ring $\mathrm{E}$ of $\mathbf{3 1}$ (six-membered ring) compared to compounds 34 and 35 (five-membered rings) slightly changed the docking arrangement (cf. Figure 4d) and caused reduced hydrophobic interactions in particular with W79. 


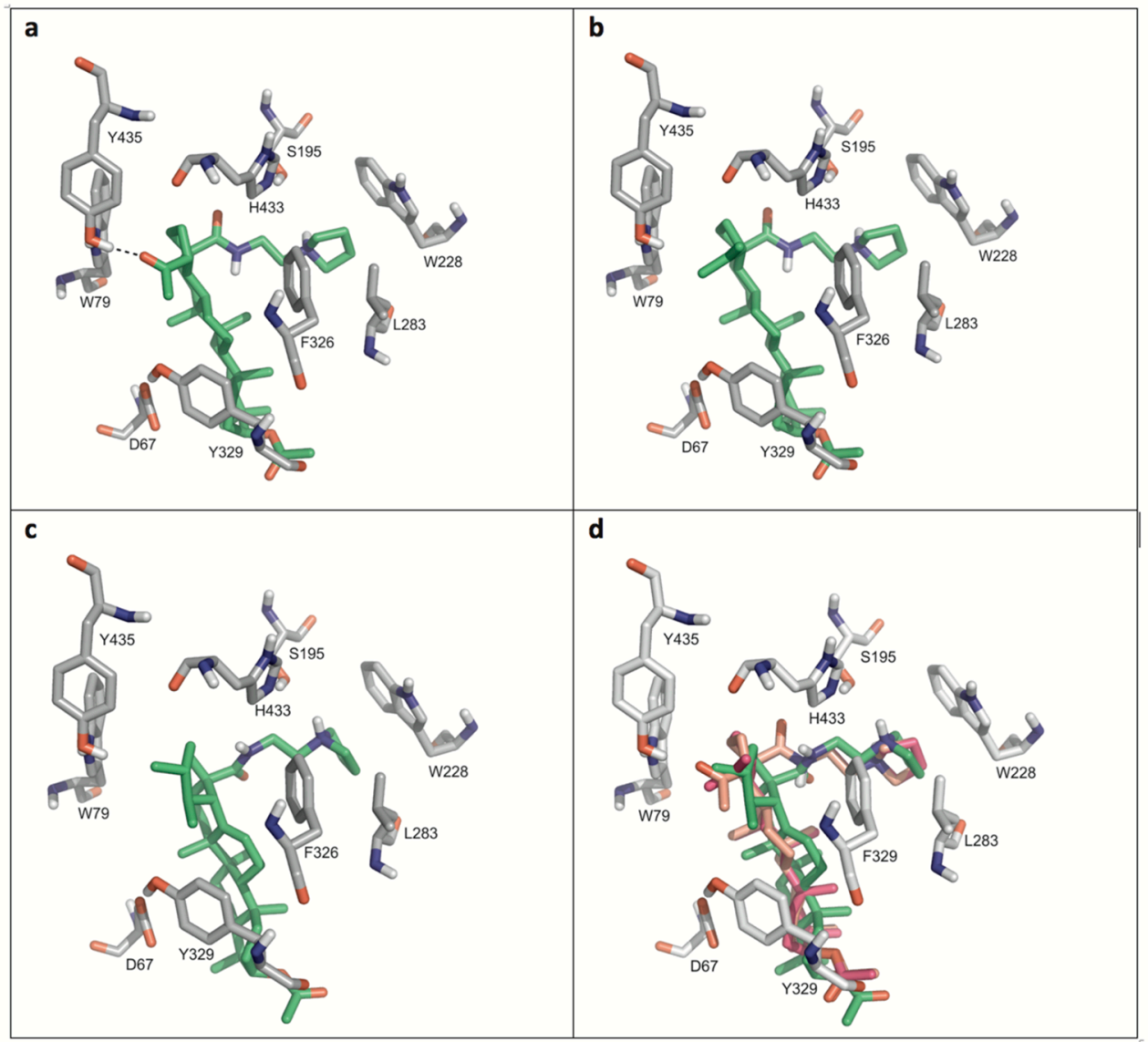

Figure 4. Details of the most favored docking arrangements of 35 (a), 34 (b) and 31 (c). (d) An overlay of all three ligands (orange colored: carbon atoms of 35; magenta colored: carbon atoms of 34; green colored: carbon atoms of 31). Hydrophobic interactions with the side chains of W79, W228, L283, Y329, and F326 preferentially stabilize the docking poses. As for 35 (a) a hydrogen bond with Y435 is formed which might explain the higher affinity compared to the other compounds.

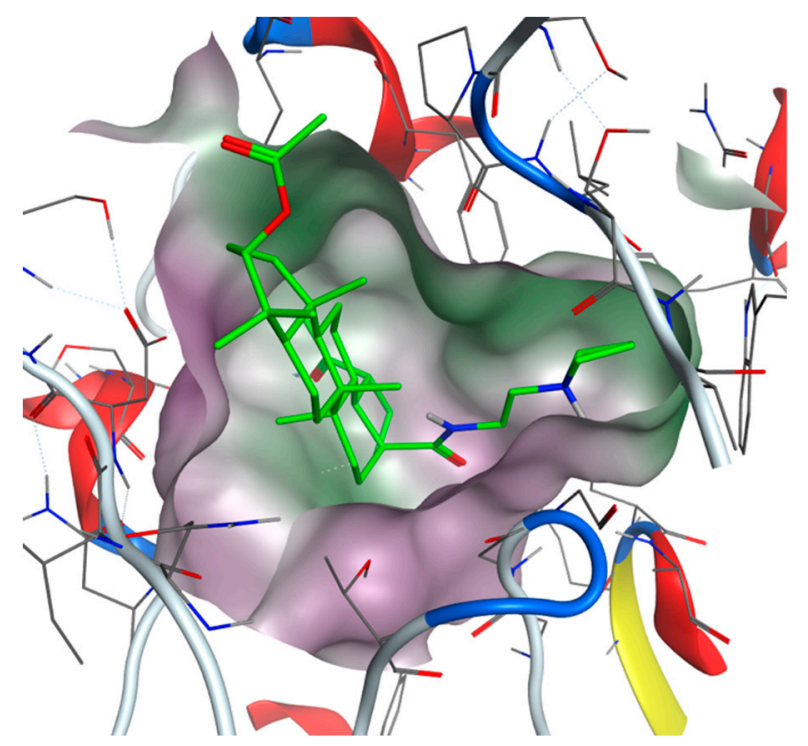

Figure 5. View from the top to the binding site of $\mathrm{BChE}$ with bound compound 35 (green colored: carbon atoms) with displayed lipophilic potential (green surface). The more hydrophilic site is colored magenta. 


\section{Materials and Methods}

\subsection{General}

All chemicals, reagents and technical equipment were purchased in Germany unless otherwise stated. NMR spectra were recorded using the Varian spectrometers Gemini 2000 or Unity 500 ( $\delta$ given in ppm, $J$ in Hz; typical experiments: H-H-COSY, HMBC, HSQC, NOESY), MS spectra were taken on a Finnigan MAT LCQ 7000 (electrospray, voltage $4.1 \mathrm{kV}$, sheath gas nitrogen) instrument. The optical rotations were measured on a Perkin-Elmer polarimeter at $20{ }^{\circ} \mathrm{C}$; TLC was performed on silica gel (Merck 5554, detection with cerium molybdate reagent) melting points were uncorrected (Leica hot stage microscope or BÜCHI Melting Point M-565) and elemental analyses were performed on a Foss-Heraeus Vario EL (CHNS) unit. IR spectra were recorded on a Perkin Elmer FT-IR spectrometer Spectrum 1000 or on a Perkin-Elmer Spectrum Two (UATR Two Unit). The solvents were dried according to usual procedures. The purity of the compounds was determined by HPLC and found to be $>96 \%$. Ursolic (1), betulinic (4), and platanic acid (5) were obtained from Betulinines (Stříbrná Skalice, Czech Republic), oleanolic acid (2) was purchased from Carbone Scientific (London, UK) and maslinic acid (3) was synthesized as previously described [20,21].

\subsection{Biology}

A TECAN SpectraFluorPlus working in the kinetic mode and measuring the absorbance at $\lambda=415 \mathrm{~nm}$ was used for the enzymatic studies. Acetylcholinesterase (from Electrophorus electricus), 5,5'-dithiobis-(2-nitrobenzoic acid) (DTNB) and acetylthiocholine iodide were purchased from Sigma. Butyrylcholinesterase (from equine serum) was purchased from Fluka. Preparation of the solutions, assay procedure, as well as molecular modeling conditions can be found in the Supplementary Materials.

\section{Experimental}

\subsection{General}

The preparation of the platanic carboxamides 15, 20, 25, 30, 35, and 40 was performed as previously described [12,22]. Betulinic and ursolic carboxamides 11, 14, 16, 19, 21, 24, 26, 29, 31, $34,36,39,41,44,46$, and 49 were prepared as previously reported in the literature [23]. Experimental procedures and full analytical data of these compounds can be found in the Supplementary Materials.

\subsection{General Procedure A for the Acetylation of Triterpenoic Acids (6-10)}

To a solution of triterpenoic acid 1-5 $(11 \mathrm{mmol})$ in dry DCM $(150 \mathrm{~mL})$ was added triethylamine $(4.6 \mathrm{~mL}, 33 \mathrm{mmol})$, acetic anhydride $(3.1 \mathrm{~mL}, 33 \mathrm{mmol})$ and DMAP (cat.). After stirring for two days at $25{ }^{\circ} \mathrm{C}$, a saturated solution of $\mathrm{NH}_{3}$ in $\mathrm{MeOH}$ was added $(3 \mathrm{~mL})$ and the mixture was stirred for another $30 \mathrm{~min}$. Dilution with DCM and subsequent aqueous work-up provided crude acetates. Recrystallization from EtOH yielded acetates 6-10 (90-96\%) as colorless solids, whose spectroscopic data were in full agreement with data from the literature.

\subsection{General Procedure B for the Synthesis of Triterpenoic Amides (11-15, 21-25, 31-35, and 41-45)}

Triterpenoic acetates 6-10 $(0.8 \mathrm{mmol})$ were each dissolved in dry DCM $(15 \mathrm{~mL})$, cooled to $0{ }^{\circ} \mathrm{C}$, then oxalyl chloride ( $3.2 \mathrm{mmol}$ ) and dry DMF (3 drops) were added. After warming to $25^{\circ} \mathrm{C}$, the mixture was stirred for $1 \mathrm{~h}$. The solvent was removed under reduced pressure, re-evaporated with dry THF $(4 \times 15 \mathrm{~mL})$, and the residue was immediately resolved in dry DCM $(10 \mathrm{~mL})$. This solution was then added dropwise to a solution of the amino compound $(2.4 \mathrm{mmol})$ in dry DCM (5 mL) and stirred at $25^{\circ} \mathrm{C}$ for $2 \mathrm{~h}$. After usual aqueous work-up, the solvent was removed under reduced pressure and the crude products were subjected to column chromatography (silica gel, chloroform/methanol mixtures). Compounds 11-15, 21-25, 31-35, and 41-45 were each obtained as colorless solids. 


\subsection{General Procedure C for the Deacetylation of Triterpenoic Amides (16-20, 26-30, 36-40, and 46-50)}

To a solution of the acetylated amide $(0.33 \mathrm{mmol})$ in methanol $(10 \mathrm{~mL})$ was added a solution of potassium hydroxide $(1.65 \mathrm{mmol})$ in methanol $(2 \mathrm{~mL})$. The mixture was stirred at $25{ }^{\circ} \mathrm{C}$ for 2 or 3 days. After completion of the reaction (as indicated by TLC), aq. $\mathrm{HCl}$ was added until pH $=7$. After usual work-up, the solvent was removed under reduced pressure and the residue was subjected to column chromatography (silica gel, chloroform/methanol mixtures) yielding compounds 16-20, 26-30, 36-40, and 46-50 each as colorless solids. (3ß)-3-Acetyloxy-urs-12-en-28-oic acid (6), Compound 6

was prepared according to general procedure A from ursolic acid (1). Yield: 96\%; m.p. $287-290{ }^{\circ} \mathrm{C}$ (lit.: $\left.289-290{ }^{\circ} \mathrm{C}[24]\right)$.

(3ß)-3-Acetyloxy-olean-12-en-28-oic acid (7), Compound 7 was prepared according to general procedure A from oleanolic acid (2). Yield: 90\%; m.p. $259-261^{\circ} \mathrm{C}$ (lit.: 255-257 ${ }^{\circ} \mathrm{C}$ [25]).

(2 $\alpha, 3 \beta)-2,3-D i a c e t y l o x y$-olean-12-en-28-oic acid (8), Compound 8 was prepared according to general procedure A from maslinic acid (3). Yield: 91\%; m.p. $172-175{ }^{\circ} \mathrm{C}\left(1 .: 170-173{ }^{\circ} \mathrm{C}\right.$ [26]).

(3ß)-3-Acetyloxy-lup-20(29)en-28-oic acid (9), Compound 9 was prepared according to general procedure A from betulinic acid (4). Yield: 93\%; m.p. $281-284{ }^{\circ} \mathrm{C}$ (lit.: 280-282 ${ }^{\circ} \mathrm{C}$ [27]).

(3ß)-3-Acetyloxy-20-oxo-30-norlupan-28-oic acid (10), Compound 10 was prepared according to general procedure A from platanic acid (5). Yield: 94\%; m.p. 256-259 ${ }^{\circ} \mathrm{C}$ (lit.: 252-255 ${ }^{\circ} \mathrm{C}$ [28]).

(3ß)-N-(2-Aminoethyl)-3-acetyloxy-olean-12-en-28-amide (12), Compound 12 was prepared from 7 according to general procedure $\mathrm{B}$ using ethylenediamine as amino compound. Column chromatography $\left(\mathrm{SiO}_{2}, \mathrm{CHCl}_{3} / \mathrm{MeOH}\right.$ 9:1) gave 12 (yield: 75\%); m.p. 212-215 ${ }^{\circ} \mathrm{C}$ (decomp.); $[\alpha]_{\mathrm{D}}=+37.8^{\circ}\left(\mathrm{c} 0.350, \mathrm{CHCl}_{3}\right) ; \mathrm{R}_{\mathrm{f}}=0.67\left(\mathrm{CHCl}_{3} / \mathrm{MeOH} / \mathrm{NH}_{4} \mathrm{OH}\right.$ 90:10:1); IR (ATR): $v=2944 \mathrm{~m}$, 1732 m, 1628 m, 1523 m, 1364 s, 1244 s, 1027 m, 985 m, 824 m, 752 m cm $^{-1}$; ${ }^{1} \mathrm{H}-\mathrm{NMR}\left(400 \mathrm{MHz}, \mathrm{CDCl}_{3}\right)$ : $\delta=7.04(\mathrm{t}, \mathrm{J}=5.5 \mathrm{~Hz}, 1 \mathrm{H}, \mathrm{NH}), 5.40(\mathrm{t}, \mathrm{J}=3.4 \mathrm{~Hz}, 1 \mathrm{H}, 12-\mathrm{H}), 4.53-4.45(\mathrm{~m}, 1 \mathrm{H}, 3-\mathrm{H}), 3.68-3.56(\mathrm{~m}, 1 \mathrm{H}$, 31- $\left.\mathrm{H}_{\mathrm{a}}\right), 3.40-3.29\left(\mathrm{~m}, 1 \mathrm{H}, 31-\mathrm{H}_{\mathrm{b}}\right), 3.24-3.11(\mathrm{~m}, 2 \mathrm{H}, 32-\mathrm{H}), 2.65(\mathrm{dd}, \mathrm{J}=12.7,4.6 \mathrm{~Hz}, 1 \mathrm{H}, 18-\mathrm{H}), 2.04$ (s, 3H, Ac), 2.01-1.82 (m, 3H, 16- $\left.\mathrm{H}_{\mathrm{a}}, 11-\mathrm{H}_{\mathrm{a}}, 11-\mathrm{H}_{\mathrm{b}}\right), 1.79-1.22\left(\mathrm{~m}, 14 \mathrm{H}, 19-\mathrm{H}_{\mathrm{a}}, 1-\mathrm{H}_{\mathrm{a}}, 2-\mathrm{H}_{\mathrm{a}}, 2-\mathrm{H}_{\mathrm{b}}, 7-\mathrm{H}_{\mathrm{a}}\right.$, $\left.7-\mathrm{H}_{\mathrm{b}}, 9-\mathrm{H}, 16-\mathrm{H}_{\mathrm{b}}, 6-\mathrm{H}_{\mathrm{a}}, 15-\mathrm{H}_{\mathrm{a}}, 22-\mathrm{H}_{\mathrm{a}}, 6-\mathrm{H}_{\mathrm{b}}, 21-\mathrm{H}_{\mathrm{a}}, 22-\mathrm{H}_{\mathrm{b}}\right), 1.22-1.11\left(\mathrm{~m}, 2 \mathrm{H}, 19-\mathrm{H}_{\mathrm{b}}, 21-\mathrm{H}_{\mathrm{b}}\right), 1.14(\mathrm{~s}, 3 \mathrm{H}$, 27-H), 1.10-0.95 (m, 2H, 1- $\left.\mathrm{H}_{\mathrm{b}}, 15-\mathrm{H}_{\mathrm{b}}\right), 0.93$ (s, 3H, 25-H), 0.90 (s, 3H, 30-H), 0.89 (s, 3H, 29-H), 0.86 (s, 3H, 23-H), 0.85 (s, 3H, 24-H), 0.84-0.79 (m, 1H, 5-H), 0.73 (s, 3H, 26-H) ppm; ${ }^{13} \mathrm{C}-\mathrm{NMR}(101 \mathrm{MHz}$, $\left.\mathrm{CDCl}_{3}\right): \delta=180.8$ (C-28), 171.0 (Ac), 144.0 (C-13), 123.1 (C-12), 80.7 (C-3), 55.2 (C-5), 47.5 (C-9), 46.6 (C-19), 46.3 (C-17), 41.8 (C-14), 41.4 (C-18), 40.4 (C-32), 39.4 (C-8), 38.2 (C-1), 38.1 (C-31), 37.7 (C-4), 36.9 (C-10), 34.2 (C-21), 33.1 (C-30), 32.3 (C-22), 32.2 (C-7), 30.6 (C-20), 28.0 (C-23), 27.2 (C-15), 25.8 (C-27), 23.6 (C-2), 23.5 (C-16), 23.5 (C-11), 23.5 (C-29), 21.3 (Ac), 18.2 (C-6), 16.9 (C-26), 16.7 (C-24), 15.5 (C-25) ppm; MS (ESI, MeOH): $\mathrm{m} / \mathrm{z}=541.3\left(100 \%,[\mathrm{M}+\mathrm{H}]^{+}\right)$; analysis calcd. for $\mathrm{C}_{34} \mathrm{H}_{56} \mathrm{~N}_{2} \mathrm{O}_{3}(540.83)$ : C 75.51, H 10.44, N 5.18; found: C 75.42, H 10.57, N 5.07.

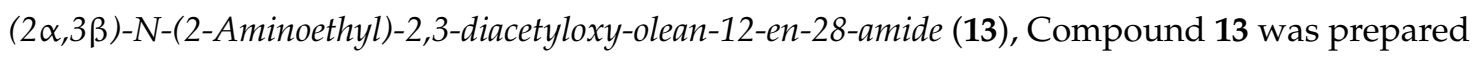
from 8 according to general procedure B using ethylenediamine as amino compound. Column chromatography $\left(\mathrm{SiO}_{2}, \mathrm{CHCl}_{3} / \mathrm{MeOH} 9: 1\right)$ gave 13 (yield: 74\%); m.p. 151-154 ${ }^{\circ} \mathrm{C} ;[\alpha]_{\mathrm{D}}=+18.7^{\circ}$ (c 0.330, $\left.\mathrm{CHCl}_{3}\right) ; \mathrm{R}_{\mathrm{f}}=0.63\left(\mathrm{CHCl}_{3} / \mathrm{MeOH} / \mathrm{NH}_{4} \mathrm{OH}\right.$ 90:10:1); IR (KBr): $v=3426$ br s, $2946 \mathrm{~s}, 1742 \mathrm{~s}$, $1636 \mathrm{~m}, 1522 \mathrm{~m}, 1458 \mathrm{~m}, 1436 \mathrm{w}, 1368 \mathrm{~m}, 1254 \mathrm{~s}, 1044 \mathrm{~m} \mathrm{~cm}^{-1} ;{ }^{1} \mathrm{H}-\mathrm{NMR}\left(400 \mathrm{MHz}, \mathrm{CDCl}_{3}\right): \delta=6.36(\mathrm{t}$, $\mathrm{J}=5.5 \mathrm{~Hz}, 1 \mathrm{H}, \mathrm{NH}), 5.37(\mathrm{t}, \mathrm{J}=3.6 \mathrm{~Hz}, 1 \mathrm{H}, 12-\mathrm{H}), 5.08(\mathrm{ddd}, \mathrm{J}=11.1,10.9,4.6 \mathrm{~Hz}, 1 \mathrm{H}, 2-\mathrm{H}), 4.73(\mathrm{~d}$, $\mathrm{J}=10.3 \mathrm{~Hz}, 1 \mathrm{H}, 3-\mathrm{H}), 3.48-3.39\left(\mathrm{~m}, 1 \mathrm{H}, 31-\mathrm{H}_{\mathrm{a}}\right), 3.12-3.02\left(\mathrm{~m}, 1 \mathrm{H}, 31-\mathrm{H}_{\mathrm{b}}\right), 2.87-2.76(\mathrm{~m}, 2 \mathrm{H}, 32-\mathrm{H})$, $2.56(\mathrm{dd}, \mathrm{J}=13.1,4.3 \mathrm{~Hz}, 1 \mathrm{H}, 18-\mathrm{H}), 2.04$ (s, 3H, Ac), 2.08-1.83 (m, 4H, 1- $\left.\mathrm{H}_{\mathrm{a}}, 16-\mathrm{H}_{\mathrm{a}}, 11-\mathrm{H}_{\mathrm{a}}, 11-\mathrm{H}_{\mathrm{b}}\right)$, $1.97(\mathrm{~s}, 3 \mathrm{H}, \mathrm{Ac}), 1.80-1.24\left(\mathrm{~m}, 11 \mathrm{H}, 19-\mathrm{H}_{\mathrm{a}}, 22-\mathrm{H}_{\mathrm{a}}, 16-\mathrm{H}_{\mathrm{b}}, 9-\mathrm{H}, 22-\mathrm{H}_{\mathrm{b}}, 6-\mathrm{H}_{\mathrm{a}}, 15-\mathrm{H}_{\mathrm{a}}, 7-\mathrm{H}_{\mathrm{a}}, 6-\mathrm{H}_{\mathrm{b}}, 21-\mathrm{H}_{\mathrm{a}}\right.$, 7- $\left.\mathrm{H}_{\mathrm{b}}\right), 1.23-1.09\left(\mathrm{~m}, 2 \mathrm{H}, 21-\mathrm{H}_{\mathrm{b}}, 19-\mathrm{H}_{\mathrm{b}}\right), 1.14(\mathrm{~s}, 3 \mathrm{H}, 27-\mathrm{H}), 1.11-0.99\left(\mathrm{~m}, 2 \mathrm{H}, 1-\mathrm{H}_{\mathrm{b}}, 15-\mathrm{H}_{\mathrm{b}}\right), 1.04(\mathrm{~s}, 3 \mathrm{H}$, 25-H), 0.99-0.92 (m, 1H, 5-H), 0.90 (s, 9H, 24-H, 29-H, 30-H), 0.89 (s, 3H, 23-H), 0.76 (s, 3H, 26-H) ppm; ${ }^{13} \mathrm{C}-\mathrm{NMR}\left(101 \mathrm{MHz}, \mathrm{CDCl}_{3}\right): \delta=178.8$ (C-28), 170.9 (Ac), 170.7 (Ac), 144.9 (C-13), 122.5 (C-12), 80.7 (C-3), 70.1 (C-2), 54.9 (C-5), 47.6 (C-9), 46.8 (C-19), 46.5 (C-17), 44.0 (C-1), 42.3 (C-18), 42.2 (C-14), 41.8 
(C-31), 41.4 (C-32), 39.6 (C-8), 39.5 (C-4), 38.2 (C-10), 34.3 (C-21), 33.1 (C-30), 32.9 (C-22), 32.3 (C-7), 30.9 (C-20), 28.5 (C-23), 27.4 (C-15), 25.9 (C-27), 23.8 (C-16), 23.8 (C-29), 23.7 (C-11), 21.3 (Ac), 21.0 (Ac), 18.3 (C-6), 17.8 (C-24), 17.1 (C-26), 16.6 (C-25) ppm; MS (ESI, MeOH): $\left.\mathrm{m} / \mathrm{z}=599(100 \% \text {, [M + H] }]^{+}\right)$; analysis calcd. for $\mathrm{C}_{36} \mathrm{H}_{58} \mathrm{~N}_{2} \mathrm{O}_{5}$ (598.87): C 72.20, $\mathrm{H}$ 9.76, N 4.68; found: C 72.01, H 9.92, N 4.44.

(3ß)-N-(2-Aminoethyl)-3-hydroxy-olean-12-en-28-amide (17), Compound 17 was prepared from 12 according to general procedure $\mathrm{C}$. Column chromatography $\left(\mathrm{SiO}_{2}, \mathrm{CHCl}_{3} / \mathrm{MeOH} / \mathrm{NH}_{4} \mathrm{OH}\right.$ 90:10:0.1) gave 17 (yield: 80\%); m.p. $221-224{ }^{\circ} \mathrm{C}$ (decomp.); $[\alpha]_{\mathrm{D}}=+55.3^{\circ}\left(\mathrm{c} 0.315, \mathrm{CHCl}_{3}\right) ; \mathrm{R}_{\mathrm{f}}=0.61$ $\left(\mathrm{CHCl}_{3} / \mathrm{MeOH} / \mathrm{NH}_{4} \mathrm{OH}\right.$ 90:10:1); IR (ATR): $v=3374 \mathrm{w}, 2941 \mathrm{~m}, 1622 \mathrm{~m}, 1530 \mathrm{~m}, 1456 \mathrm{~m}, 1387 \mathrm{~s}, 1361 \mathrm{~s}$, $1344 \mathrm{~s}, 1322 \mathrm{~s}, 1187 \mathrm{~m}, 1137 \mathrm{~m}, 1097 \mathrm{~m}, 1023 \mathrm{~m}, 996 \mathrm{~m}, 825 \mathrm{~m} \mathrm{~cm}^{-1} ;{ }^{1} \mathrm{H}-\mathrm{NMR}\left(500 \mathrm{MHz}, \mathrm{CD}_{3} \mathrm{OD}\right): \delta=5.36$ $(\mathrm{t}, \mathrm{J}=3.7 \mathrm{~Hz}, 1 \mathrm{H}, 12-\mathrm{H}), 3.50-3.43\left(\mathrm{~m}, 1 \mathrm{H}, 31-\mathrm{H}_{\mathrm{a}}\right), 3.37-3.32\left(\mathrm{~m}, 1 \mathrm{H}, 31-\mathrm{H}_{\mathrm{b}}\right), 3.15(\mathrm{dd}, \mathrm{J}=11.3,4.8 \mathrm{~Hz}$, $1 \mathrm{H}, 3-\mathrm{H}), 3.07-2.95(\mathrm{~m}, 2 \mathrm{H}, 32-\mathrm{H}), 2.79(\mathrm{dd}, \mathrm{J}=13.2,4.4 \mathrm{~Hz}, 1 \mathrm{H}, 18-\mathrm{H}), 2.10(\mathrm{ddd}, \mathrm{J}=13.8,13.0,4.0 \mathrm{~Hz}$, $\left.1 \mathrm{H}, 16-\mathrm{H}_{\mathrm{a}}\right), 1.99-1.86\left(\mathrm{~m}, 2 \mathrm{H}, 11-\mathrm{H}_{\mathrm{a}}, 11-\mathrm{H}_{\mathrm{b}}\right), 1.83-1.74\left(\mathrm{~m}, 1 \mathrm{H}, 19-\mathrm{H}_{\mathrm{a}}\right), 1.70-1.35\left(\mathrm{~m}, 12 \mathrm{H}, 22-\mathrm{H}_{\mathrm{a}}, 1-\mathrm{H}_{\mathrm{a}}\right.$, 9-H, 15- $\left.\mathrm{H}_{\mathrm{a}}, 2-\mathrm{H}_{\mathrm{a}}, 2-\mathrm{H}_{\mathrm{b}}, 16-\mathrm{H}_{\mathrm{b}}, 6-\mathrm{H}_{\mathrm{a}}, 22-\mathrm{H}_{\mathrm{b}}, 7-\mathrm{H}_{\mathrm{a}}, 6-\mathrm{H}_{\mathrm{b}}, 21-\mathrm{H}_{\mathrm{a}}\right), 1.33-1.27\left(\mathrm{~m}, 1 \mathrm{H}, 7-\mathrm{H}_{\mathrm{b}}\right), 1.25-1.14(\mathrm{~m}$, $\left.2 \mathrm{H}, 21-\mathrm{H}_{\mathrm{b}}, 19-\mathrm{H}_{\mathrm{b}}\right), 1.18(\mathrm{~s}, 3 \mathrm{H}, 27-\mathrm{H}), 1.11-0.99\left(\mathrm{~m}, 2 \mathrm{H}, 15-\mathrm{H}_{\mathrm{b}}, 1-\mathrm{H}_{\mathrm{b}}\right), 0.97(\mathrm{~s}, 3 \mathrm{H}, 23-\mathrm{H}), 0.96(\mathrm{~s}, 3 \mathrm{H}$, 29-H), 0.94 (s, 3H, 25-H), 0.92 (s, 3H, 30-H), 0.79 (s, 3H, 26-H), 0.78 (s, 3H, 24-H), 0.77-0.74 (m, 1H, 5-H) ppm; ${ }^{13} \mathrm{C}-\mathrm{NMR}\left(126 \mathrm{MHz}, \mathrm{CD}_{3} \mathrm{OD}\right): \delta=182.0$ (C-28), 145.0 (C-13), 124.2 (C-12), 79.6 (C-3), 56.7 (C-5), 49.0 (C-9), 47.6 (C-17), 47.6 (C-19), 42.9 (C-14), 42.4 (C-18), 40.8 (C-32), 40.7 (C-8), 39.8 (C-4), 39.8 (C-1), 38.8 (C-31), 38.1 (C-10), 35.0 (C-21), 34.2 (C-22), 33.8 (C-7), 33.5 (C-30), 31.6 (C-20), 28.7 (C-23), 28.5 (C-15), 27.8 (C-2), 26.5 (C-27), 24.5 (C-11), 24.0 (C-29), 24.0 (C-16), 19.5 (C-6), 17.9 (C-26), 16.3 (C-24), 15.9 (C-25) ppm; MS (ESI, MeOH): $\left.\mathrm{m} / \mathrm{z}=499.3(100 \% \text {, [M + H] }]^{+}\right)$; analysis calcd. for $\mathrm{C}_{32} \mathrm{H}_{54} \mathrm{~N}_{2} \mathrm{O}_{2}$ (498.80): C 77.06, H 10.91, N 5.62; found: C 76.90, H 11.05, N 5.41.

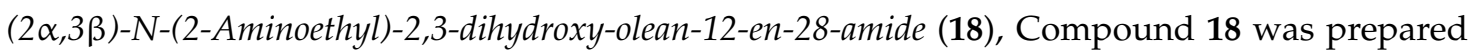
from 13 according to general procedure $\mathrm{C}$. Column chromatography $\left(\mathrm{SiO}_{2}, \mathrm{CHCl}_{3} / \mathrm{MeOH}\right.$ 9:1) gave 18 (yield: $60 \%$ ); m.p. $260-266{ }^{\circ} \mathrm{C}$ (decompn.); $[\alpha]_{\mathrm{D}}=+47.6^{\circ}$ (c $\left.0.335, \mathrm{CHCl}_{3}\right) ; \mathrm{R}_{\mathrm{f}}=0.36$ $\left(\mathrm{CHCl}_{3} / \mathrm{MeOH} / \mathrm{NH}_{4} \mathrm{OH} 90: 10: 1\right) ; \mathrm{IR}(\mathrm{KBr}): v=3424$ br s, $944 \mathrm{~m}, 1636 \mathrm{~m}, 1528 \mathrm{~m}, 1460 \mathrm{w}, 1384 \mathrm{w}$, 1166w, $1050 \mathrm{~m} \mathrm{~cm}^{-1} ;{ }^{1} \mathrm{H}-\mathrm{NMR}\left(400 \mathrm{MHz}, \mathrm{CD}_{3} \mathrm{OD}\right): \delta=5.36(\mathrm{t}, \mathrm{J}=3.7 \mathrm{~Hz}, 1 \mathrm{H}, 12-\mathrm{H}), 3.61(\mathrm{ddd}, \mathrm{J}=11.3$, 9.5, 4.5 Hz, 1H, 2-H), 3.43-3.35 (m, 1H, 31- $\left.\mathrm{H}_{\mathrm{a}}\right), 3.29-3.22\left(\mathrm{~m}, 1 \mathrm{H}, 31-\mathrm{H}_{\mathrm{b}}\right), 2.90(\mathrm{~d}, \mathrm{~J}=9.6 \mathrm{~Hz}, 1 \mathrm{H}, 3-\mathrm{H})$, $2.89(\mathrm{t}, \mathrm{J}=6.5 \mathrm{~Hz}, 2 \mathrm{H}, 32-\mathrm{H}), 2.81(\mathrm{dd}, \mathrm{J}=13.5,4.5 \mathrm{~Hz}, 1 \mathrm{H}, 18-\mathrm{H}), 2.14-2.03\left(\mathrm{~m}, 1 \mathrm{H}, 16-\mathrm{H}_{\mathrm{a}}\right), 2.03-1.88(\mathrm{~m}$, $\left.3 \mathrm{H}, 11-\mathrm{H}_{\mathrm{a}}, 11-\mathrm{H}_{\mathrm{b}}, 1-\mathrm{H}_{\mathrm{a}}\right), 1.78\left(\mathrm{t}, \mathrm{J}=13.5 \mathrm{~Hz}, 1 \mathrm{H}, 19-\mathrm{H}_{\mathrm{a}}\right), 1.70-1.26\left(\mathrm{~m}, 10 \mathrm{H}, 9-\mathrm{H}, 22-\mathrm{H}_{\mathrm{a}}, 15-\mathrm{H}_{\mathrm{a}}, 16-\mathrm{H}_{\mathrm{b}}\right.$, $\left.6-\mathrm{H}_{\mathrm{a}}, 22-\mathrm{H}_{\mathrm{b}}, 7-\mathrm{H}_{\mathrm{a}}, 6-\mathrm{H}_{\mathrm{b}}, 21-\mathrm{H}_{\mathrm{a}}, 7-\mathrm{H}_{\mathrm{b}}\right), 1.23-1.13\left(\mathrm{~m}, 2 \mathrm{H}, 19-\mathrm{H}_{\mathrm{b}}, 21-\mathrm{H}_{\mathrm{b}}\right), 1.18(\mathrm{~s}, 3 \mathrm{H}, 27-\mathrm{H}), 1.10-1.02$ $\left(\mathrm{m}, 1 \mathrm{H}, 15-\mathrm{H}_{\mathrm{b}}\right), 1.01(\mathrm{~s}, 3 \mathrm{H}, 23-\mathrm{H}), 1.00(\mathrm{~s}, 3 \mathrm{H}, 25-\mathrm{H}), 0.95(\mathrm{~s}, 3 \mathrm{H}, 30-\mathrm{H}), 0.91$ (s, 3H, 29-H), 0.93-0.89 $\left(\mathrm{m}, 1 \mathrm{H}, 1-\mathrm{H}_{\mathrm{b}}\right), 0.88-0.82(\mathrm{~m}, 1 \mathrm{H}, 5-\mathrm{H}), 0.80(\mathrm{~s}, 3 \mathrm{H}, 24-\mathrm{H}), 0.78(\mathrm{~s}, 3 \mathrm{H}, 26-\mathrm{H}) \mathrm{ppm} ;{ }^{13} \mathrm{C}-\mathrm{NMR}(101 \mathrm{MHz}$, $\left.\mathrm{CD}_{3} \mathrm{OD}\right): \delta=181.5$ (C-28), 145.2 (C-13), 123.9 (C-12), 84.4 (C-3), 69.4 (C-2), 56.6 (C-5), 48.9 (C-9), 48.1 (C-1), 47.6 (C-17), 47.5 (C-19), 42.9 (C-14), 42.5 (C-18), 41.2 (C-32), 40.7 (C-8), 40.5 (C-4), 40.4 (C-31), 39.2 (C-10), 35.1 (C-21), 34.3 (C-22), 33.7 (C-7), 33.5 (C-30), 31.6 (C-20), 29.3 (C-23), 28.5 (C-15), 26.5 (C-27), 24.6 (C-11), 24.0 (C-29), 23.9 (C-16), 19.5 (C-6), 17.9 (C-26), 17.4 (C-24), 17.1 (C-25) ppm; MS (ESI, $\mathrm{MeOH}): \mathrm{m} / \mathrm{z}=515\left(100 \%\right.$, $\left.[\mathrm{M}+\mathrm{H}]^{+}\right)$; analysis calcd. for $\mathrm{C}_{32} \mathrm{H}_{54} \mathrm{~N}_{2} \mathrm{O}_{3}(514.80): \mathrm{C} 74.66, \mathrm{H}$ 10.57, N 5.44; found: C 74.49, H 10.74, N 5.28.

(3ß)-N-[2-(Dimethylamino)-ethyl]-3-acetyloxy-olean-12-en-28-amide (22), Compound 22 was prepared from 7 according to general procedure $\mathrm{B}$ using $\mathrm{N}, \mathrm{N}$-dimethylethylene diamine as amino compound. Column chromatography $\left(\mathrm{SiO}_{2}, \mathrm{CHCl}_{3} / \mathrm{MeOH} 95: 5\right)$ gave 22 (yield: $95 \%$ ); m.p. $227-230{ }^{\circ} \mathrm{C}$ (decomp.), Lit.: $255^{\circ} \mathrm{C}$ (decomp.) [29]; $[\alpha]_{\mathrm{D}}=+53.0^{\circ}$ (c 0.320, $\mathrm{CHCl}_{3}$ ), Lit.: $+51.8^{\circ}$ (c 0.34, $\left.\mathrm{CHCl}_{3}\right)$ [29]; $\mathrm{R}_{\mathrm{f}}=0.38$ $\left(\mathrm{CHCl}_{3} / \mathrm{MeOH} 9: 1\right)$; IR (ATR): $v=2944 \mathrm{~m}, 1732 \mathrm{~m}, 1640 \mathrm{w}, 1464 \mathrm{~m}, 1365 \mathrm{~m}, 1244 \mathrm{~s}, 1027 \mathrm{~m}, 986 \mathrm{~m}$, $753 \mathrm{~m} \mathrm{~cm}^{-1} ;{ }^{1} \mathrm{H}-\mathrm{NMR}\left(400 \mathrm{MHz}, \mathrm{CDCl}_{3}\right): \delta=6.77(\mathrm{t}, \mathrm{J}=5.3 \mathrm{~Hz}, 1 \mathrm{H}, \mathrm{NH}), 5.38(\mathrm{t}, \mathrm{J}=3.6 \mathrm{~Hz}, 1 \mathrm{H}$, 12-H), 4.51-4.45 (m, 1H, 3-H), 3.61-3.51 (m, 1H, 31- $\left.\mathrm{H}_{\mathrm{a}}\right), 3.37-3.27\left(\mathrm{~m}, 1 \mathrm{H}, 31-\mathrm{H}_{\mathrm{b}}\right), 2.89-2.83(\mathrm{~m}, 2 \mathrm{H}$, 32-H), 2.65-2.56 (m, 1H, 18-H), $2.61\left(\mathrm{~s}, 6 \mathrm{H}, 33-\mathrm{H}, 33^{\prime}-\mathrm{H}\right), 2.03(\mathrm{~s}, 3 \mathrm{H}, \mathrm{Ac}), 2.02-1.93\left(\mathrm{~m}, 1 \mathrm{H}, 16-\mathrm{H}_{\mathrm{a}}\right)$, 1.93-1.85 (m, 2H, 11- $\left.\mathrm{H}_{\mathrm{a}}, 11-\mathrm{H}_{\mathrm{b}}\right), 1.72\left(\mathrm{t}, \mathrm{J}=13.4 \mathrm{~Hz}, 1 \mathrm{H}, 19-\mathrm{H}_{\mathrm{a}}\right), 1.66-1.22\left(\mathrm{~m}, 13 \mathrm{H}, 22-\mathrm{H}_{\mathrm{a}}, 1-\mathrm{H}_{\mathrm{a}}, 2-\mathrm{H}_{\mathrm{a}}\right.$, $\left.2-\mathrm{H}_{\mathrm{b}}, 16-\mathrm{H}_{\mathrm{b}}, 9-\mathrm{H}, 22-\mathrm{H}_{\mathrm{b}}, 6-\mathrm{H}_{\mathrm{a}}, 15-\mathrm{H}_{\mathrm{a}}, 7-\mathrm{H}_{\mathrm{a}}, 6-\mathrm{H}_{\mathrm{b}}, 21-\mathrm{H}_{\mathrm{a}}, 7-\mathrm{H}_{\mathrm{b}}\right), 1.21-1.14\left(\mathrm{~m}, 2 \mathrm{H}, 19-\mathrm{H}_{\mathrm{b}}, 21-\mathrm{H}_{\mathrm{b}}\right), 1.14$ $(\mathrm{s}, 3 \mathrm{H}, 27-\mathrm{H}), 1.10-0.97\left(\mathrm{~m}, 2 \mathrm{H}, 1-\mathrm{H}_{\mathrm{b}}, 15-\mathrm{H}_{\mathrm{b}}\right), 0.92(\mathrm{~s}, 3 \mathrm{H}, 25-\mathrm{H}), 0.90(\mathrm{~s}, 3 \mathrm{H}, 29-\mathrm{H}), 0.89(\mathrm{~s}, 3 \mathrm{H}, 30-\mathrm{H})$, 
0.85 (s, 3H, 23-H), 0.84 (s, 3H, 24-H), 0.83-0.79 (m, 1H, 5-H), 0.74 (s, 3H, 26-H) ppm; ${ }^{13} \mathrm{C}-\mathrm{NMR}(101$ $\mathrm{MHz}, \mathrm{CDCl}_{3}$ ): $\delta=179.3$ (C-28), 171.1 (Ac), 144.2 (C-13), 123.1 (C-12), 81.0 (C-3), 57.8 (C-32), 55.4 (C-5), 47.6 (C-9), 46.6 (C-19), 46.5 (C-17), 44.7 (C-33, C-33'), 42.0 (C-14), 41.9 (C-18), 39.6 (C-8), 38.3 (C-1), 37.8 (C-4), 37.0 (C-10), 36.1 (C-31), 34.3 (C-21), 33.2 (C-30), 32.9 (C-22), 32.5 (C-7), 30.8 (C-20), 28.2 (C-23), 27.5 (C-15), 25.9 (C-27), 23.7 (C-29), 23.6 (C-16, C-2, C-11), 21.4 (Ac), 18.3 (C-6), 17.1 (C-24), 16.8 (C-26), $15.6(\mathrm{C}-25)$ ppm; MS (ESI, MeOH): $\mathrm{m} / \mathrm{z}=569.5\left(100 \%,[\mathrm{M}+\mathrm{H}]^{+}\right)$; analysis calcd. for $\mathrm{C}_{36} \mathrm{H}_{60} \mathrm{~N}_{2} \mathrm{O}_{3}$ (568.89): C 76.01, H 10.63, N 4.92; found: C 75.86, H 10.83, N 4.77.

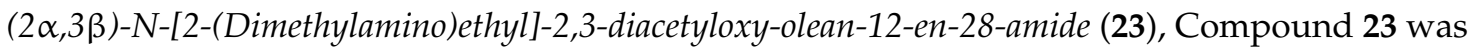
prepared from 8 according to general procedure $\mathrm{B}$ using $\mathrm{N}, \mathrm{N}$-dimethylethylene diamine as amino compound. Column chromatography $\left(\mathrm{SiO}_{2}, \mathrm{CHCl}_{3} / \mathrm{MeOH} 9: 1\right.$ ) gave 23 (yield: 94\%); m.p. 131-136 ${ }^{\circ} \mathrm{C}$; $[\alpha]_{\mathrm{D}}=+17.2^{\circ}\left(\mathrm{c} 0.340, \mathrm{CHCl}_{3}\right) ; \mathrm{R}_{\mathrm{f}}=0.31\left(\mathrm{CHCl}_{3} / \mathrm{MeOH} 95: 5\right) ; \mathrm{IR}(\mathrm{KBr}): v=3426 \mathrm{br} \mathrm{s}, 2946 \mathrm{~s}, 2772 \mathrm{w}$, 1744s, 1654m, 1512m, 1462m, 1368m, 1252s, $1156 \mathrm{w}, 1044 \mathrm{~m} \mathrm{~cm}^{-1} ;{ }^{1} \mathrm{H}-\mathrm{NMR}\left(400 \mathrm{MHz}, \mathrm{CDCl}_{3}\right): \delta=6.51$ $(\mathrm{t}, \mathrm{J}=4.4 \mathrm{~Hz}, 1 \mathrm{H}, \mathrm{NH}), 5.33(\mathrm{t}, \mathrm{J}=3.6 \mathrm{~Hz}, 1 \mathrm{H}, 12-\mathrm{H}), 5.08(\mathrm{ddd}, \mathrm{J}=11.2,10.3,4.6 \mathrm{~Hz}, 1 \mathrm{H}, 2-\mathrm{H}), 4.75(\mathrm{~d}$, $\mathrm{J}=10.3 \mathrm{~Hz}, 1 \mathrm{H}, 3-\mathrm{H}), 3.39-3.29\left(\mathrm{~m}, 1 \mathrm{H}, 31-\mathrm{H}_{\mathrm{a}}\right), 3.18-3.09\left(\mathrm{~m}, 1 \mathrm{H}, 31-\mathrm{H}_{\mathrm{b}}\right), 2.56-2.48(\mathrm{~m}, 1 \mathrm{H}, 18-\mathrm{H}), 2.36$ $(\mathrm{t}, \mathrm{J}=6.0 \mathrm{~Hz}, 2 \mathrm{H}, 32-\mathrm{H}), 2.21\left(\mathrm{~s}, 6 \mathrm{H}, 33-\mathrm{H}, 33^{\prime}-\mathrm{H}\right), 2.05(\mathrm{~s}, 3 \mathrm{H} . \mathrm{Ac}), 2.04-1.99\left(\mathrm{~m}, 1 \mathrm{H}, 1-\mathrm{H}_{\mathrm{a}}\right), 1.97(\mathrm{~s}, 3 \mathrm{H}$, Ac), $1.96-1.87\left(\mathrm{~m}, 3 \mathrm{H}, 16-\mathrm{H}_{\mathrm{a}}, 11-\mathrm{H}_{\mathrm{a}}, 11-\mathrm{H}_{\mathrm{b}}\right), 1.81-1.25\left(\mathrm{~m}, 11 \mathrm{H}, 19-\mathrm{H}_{\mathrm{a}}, 22-\mathrm{H}_{\mathrm{a}}, 16-\mathrm{H}_{\mathrm{b}}, 9-\mathrm{H}, 22-\mathrm{H}_{\mathrm{b}}, 15-\mathrm{H}_{\mathrm{a}}\right.$, 6- $\left.\mathrm{H}_{\mathrm{a}}, 7-\mathrm{H}_{\mathrm{a}}, 6-\mathrm{H}_{\mathrm{b}}, 21-\mathrm{H}_{\mathrm{a}}, 7-\mathrm{H}_{\mathrm{b}}\right), 1.22-1.12\left(\mathrm{~m}, 2 \mathrm{H}, 21-\mathrm{H}_{\mathrm{b}}, 19-\mathrm{H}_{\mathrm{b}}\right), 1.14(\mathrm{~s}, 3 \mathrm{H}, 27-\mathrm{H}), 1.05(\mathrm{~s}, 3 \mathrm{H}, 25-\mathrm{H})$, 1.10-0.99 (m, 2H, 1- $\left.\mathrm{H}_{\mathrm{b}}, 15-\mathrm{H}_{\mathrm{b}}\right), 0.99-0.94(\mathrm{~m}, 1 \mathrm{H}, 5-\mathrm{H}), 0.90$ (s, 9H, 24-H, 29-H, 30-H), 0.89 (s, 3H, 23-H), 0.79 (s, 3H, 26-H) ppm; ${ }^{13} \mathrm{C}-\mathrm{NMR}\left(101 \mathrm{MHz}, \mathrm{CDCl}_{3}\right): \delta=178.1$ (C-28), 170.9 (Ac), 170.7 (Ac), 144.7 (C-13), 122.5 (C-12), 80.7 (C-3), 70.2 (C-2), 57.7 (C-32), 55.0 (C-5), 47.6 (C-9), 46.8 (C-19), 46.5 (C-17), 45.4 (C-33, C-33'), 44.1 (C-1), 42.5 (C-18), 42.2 (C-14), 39.6 (C-8), 39.5 (C-4), 38.2 (C-10), 37.0 (C-31), 34.3 (C-21), 33.2 (C-30), 32.8 (C-22), 32.5 (C-7), 30.9 (C-20), 28.6 (C-23), 27.5 (C-15), 25.8 (C-27), 23.8 (C-16), 23.7 (C-11), 23.7 (C-29), 21.3 (Ac), 21.0 (Ac), 18.4 (C-6), 17.8 (C-24), 17.0 (C-26), 16.7 (C-25) ppm; MS (ESI, $\mathrm{MeOH}): \mathrm{m} / \mathrm{z}=627\left(100 \%,[\mathrm{M}+\mathrm{H}]^{+}\right)$; analysis calcd. for $\mathrm{C}_{38} \mathrm{H}_{62} \mathrm{~N}_{2} \mathrm{O}_{5}$ (626.92): C 72.80, $\mathrm{H}$ 9.97, N 4.47; found: C 72.63, H 10.14, N4.29.

(3ß)-N-[2-(Dimethylamino)-ethyl]-3-hydroxy-olean-12-en-28-amide (27), Compound 27 was prepared from 22 according to general procedure $\mathrm{C}$. Column chromatography $\left(\mathrm{SiO}_{2}, \mathrm{CHCl}_{3} / \mathrm{MeOH} 95: 5\right)$ gave 27 (yield: 85\%); m.p. $187-190^{\circ} \mathrm{C} ;[\alpha]_{\mathrm{D}}=+42.6^{\circ}$ (c 0.320, $\left.\mathrm{MeOH}\right) ; \mathrm{R}_{\mathrm{f}}=0.29\left(\mathrm{CHCl}_{3} / \mathrm{MeOH} 9: 1\right) ; \mathrm{IR}$ (ATR): $v=3383 b r \mathrm{w}, 2943 \mathrm{~m} ; 1624 \mathrm{~m}, 1532 \mathrm{~m}, 1431 \mathrm{~s}, 1387 \mathrm{~s}, 1319 \mathrm{~s}, 1186 \mathrm{~m}, 1032 \mathrm{~m}, 996 \mathrm{~m}, 823 \mathrm{~m}, 752 \mathrm{~s} \mathrm{~cm}^{-1}$; ${ }^{1} \mathrm{H}-\mathrm{NMR}\left(500 \mathrm{MHz}, \mathrm{CDCl}_{3}\right): \delta=6.73(\mathrm{t}, \mathrm{J}=5.2 \mathrm{~Hz}, 1 \mathrm{H}, \mathrm{NH}), 5.40(\mathrm{t}, \mathrm{J}=3.6 \mathrm{~Hz}, 1 \mathrm{H}, 12-\mathrm{H}), 3.63-3.53$ $\left(\mathrm{m}, 1 \mathrm{H}, 31-\mathrm{H}_{\mathrm{a}}\right), 3.35-3.27\left(\mathrm{~m}, 1 \mathrm{H}, 31-\mathrm{H}_{\mathrm{b}}\right), 3.21(\mathrm{dd}, \mathrm{J}=11.4,4.3 \mathrm{~Hz}, 1 \mathrm{H}, 3-\mathrm{H}), 2.87-2.81(\mathrm{~m}, 2 \mathrm{H}, 32-\mathrm{H})$, 2.64-2.57 (m, 1H, 18-H), $2.60\left(\mathrm{~s}, 6 \mathrm{H}, 33-\mathrm{H}, 33^{\prime}-\mathrm{H}\right), 1.99\left(\mathrm{ddd}, \mathrm{J}=13.7,13.6,3.9 \mathrm{~Hz}, 1 \mathrm{H}, 16-\mathrm{H}_{\mathrm{a}}\right), 1.93-1.89$ $\left(\mathrm{m}, 2 \mathrm{H}, 11-\mathrm{H}_{\mathrm{a}}, 11-\mathrm{H}_{\mathrm{b}}\right), 1.73\left(\mathrm{t}, \mathrm{J}=13.4 \mathrm{~Hz}, 1 \mathrm{H}, 19-\mathrm{H}_{\mathrm{a}}\right), 1.68-1.30\left(\mathrm{~m}, 12 \mathrm{H}, 22-\mathrm{H}_{\mathrm{a}}, 16-\mathrm{H}_{\mathrm{b}}, 1-\mathrm{H}_{\mathrm{a}}, 2-\mathrm{H}_{\mathrm{a}}\right.$, $\left.2-\mathrm{H}_{\mathrm{b}}, 9-\mathrm{H}, 22-\mathrm{H}_{\mathrm{b}}, 6-\mathrm{H}_{\mathrm{a}}, 15-\mathrm{H}_{\mathrm{a}}, 7-\mathrm{H}_{\mathrm{a}}, 6-\mathrm{H}_{\mathrm{b}}, 21-\mathrm{H}_{\mathrm{a}}\right), 1.26\left(\mathrm{ddd}, \mathrm{J}=12.3,3.0,2.9 \mathrm{~Hz}, 1 \mathrm{H}, 7-\mathrm{H}_{\mathrm{b}}\right), 1.22-1.14$ $\left(\mathrm{m}, 2 \mathrm{H}, 21-\mathrm{H}_{\mathrm{b}}, 19-\mathrm{H}_{\mathrm{b}}\right), 1.15(\mathrm{~s}, 3 \mathrm{H}, 27-\mathrm{H}), 1.05\left(\mathrm{ddd}, \mathrm{J}=14.1,3.6,3.5 \mathrm{~Hz}, 1 \mathrm{H}, 15-\mathrm{H}_{\mathrm{b}}\right), 0.98(\mathrm{~s}, 3 \mathrm{H}, 23-\mathrm{H})$, 0.98-0.93 (m, 1H, 1- $\left.\mathrm{H}_{\mathrm{b}}\right), 0.92(\mathrm{~s}, 3 \mathrm{H}, 29-\mathrm{H}), 0.90(\mathrm{~s}, 3 \mathrm{H}, 25-\mathrm{H}), 0.90(\mathrm{~s}, 3 \mathrm{H}, 30-\mathrm{H}), 0.78(\mathrm{~s}, 3 \mathrm{H}, 24-\mathrm{H}), 0.75$ (s, 3H, 26-H), 0.74-0.71 (m, 1H, 5-H) ppm; ${ }^{13} \mathrm{C}-\mathrm{NMR}\left(126 \mathrm{MHz}, \mathrm{CDCl}_{3}\right): \delta=179.2(\mathrm{C}-28), 144.2(\mathrm{C}-13)$, 123.2 (C-12), 79.1 (C-3), 57.8 (C-32), 55.3 (C-5), 47.7 (C-9), 46.7 (C-19), 46.6 (C-17), 44.7 (C-33, C-33'), 42.1 (C-14), 42.0 (C-18), 39.6 (C-8), 38.9 (C-4), 38.6 (C-1), 37.1 (C-10), 36.0 (C-31), 34.3 (C-21), 33.2 (C-30), 32.9 (C-22), 32.6 (C-7), 30.8 (C-20), 28.2 (C-23), 27.5 (C-15), 27.3 (C-2), 25.9 (C-27), 23.7 (C-16), 23.7 (C-29), 23.6 (C-11), 18.5 (C-6), 17.2 (C-26), 15.7 (C-24), 15.5 (C-25) ppm; MS (ESI, MeOH): m/z = $527.4(100 \%$, $[\mathrm{M}+\mathrm{H}]^{+}$); analysis calcd. for $\mathrm{C}_{34} \mathrm{H}_{58} \mathrm{~N}_{2} \mathrm{O}_{2}$ (526.85): C 77.51, H 11.10, N 5.32; found: C 77.37, $\mathrm{H}$ 11.31, N 5.16 .

(2 $\alpha, 3 \beta)-N$-[2-(Dimethylamino)-ethyl]-2,3-dihydroxy-olean-12-en-28-amide (28), Compound 28 was prepared from 23 according to general procedure $\mathrm{C}$. Column chromatography $\left(\mathrm{SiO}_{2}, \mathrm{CHCl}_{3} / \mathrm{MeOH}\right.$ 9:1) gave 28 (yield: $66 \%$ ); m.p. $146-149^{\circ} \mathrm{C} ;[\alpha]_{\mathrm{D}}=+43.1^{\circ}\left(\mathrm{c} 0.355, \mathrm{CHCl}_{3}\right) ; \mathrm{R}_{\mathrm{f}}=0.10\left(\mathrm{CHCl}_{3} / \mathrm{MeOH}\right.$ 95:5); IR (KBr): $v=3418 \mathrm{br} s, 2946 \mathrm{~s}, 2862 \mathrm{~s}, 2826 \mathrm{~m}, 2778 \mathrm{~m}, 1636 \mathrm{~s}, 1522 \mathrm{~m}, 1462 \mathrm{~s}, 1386 \mathrm{~m}, 1364 \mathrm{~m}, 1266 \mathrm{w}$, 1188w, 1158w, 1098w, 1050s cm ${ }^{-1} ;{ }^{1} \mathrm{H}-\mathrm{NMR}\left(400 \mathrm{MHz}, \mathrm{CDCl}_{3}\right): \delta=6.56(\mathrm{t}, \mathrm{J}=4.9 \mathrm{~Hz}, 1 \mathrm{H}, \mathrm{NH}), 5.35$ $(\mathrm{t}, \mathrm{J}=3.6 \mathrm{~Hz}, 1 \mathrm{H}, 12-\mathrm{H}), 3.68(\mathrm{ddd}, \mathrm{J}=11.3,9.5,4.5 \mathrm{~Hz}, 1 \mathrm{H}, 2-\mathrm{H}), 3.42-3.32\left(\mathrm{~m}, 1 \mathrm{H}, 31-\mathrm{H}_{\mathrm{a}}\right), 3.20-3.11$ 
$\left(\mathrm{m}, 1 \mathrm{H}, 31-\mathrm{H}_{\mathrm{b}}\right), 2.99(\mathrm{~d}, \mathrm{~J}=9.5 \mathrm{~Hz}, 1 \mathrm{H}, 3-\mathrm{H}), 2.57-2.50(\mathrm{~m}, 1 \mathrm{H}, 18-\mathrm{H}), 2.40(\mathrm{t}, \mathrm{J}=6.1 \mathrm{~Hz}, 2 \mathrm{H}, 32-\mathrm{H}), 2.25$ (s, 6H, 33-H, 33'-H), 2.01-1.90 (m, 4H, 1- $\left.\mathrm{H}_{\mathrm{a}}, 16-\mathrm{H}_{\mathrm{a}}, 11-\mathrm{H}_{\mathrm{a}}, 11-\mathrm{H}_{\mathrm{b}}\right), 1.80-1.24\left(\mathrm{~m}, 11 \mathrm{H}, 19-\mathrm{H}_{\mathrm{a}}, 22-\mathrm{H}_{\mathrm{a}}\right.$, $\left.16-\mathrm{H}_{\mathrm{b}}, 9-\mathrm{H}, 22-\mathrm{H}_{\mathrm{b}}, 15-\mathrm{H}_{\mathrm{a}}, 6-\mathrm{H}_{\mathrm{a}}, 7-\mathrm{H}_{\mathrm{a}}, 6-\mathrm{H}_{\mathrm{b}}, 21-\mathrm{H}_{\mathrm{a}}, 7-\mathrm{H}_{\mathrm{b}}\right), 1.22-1.16\left(\mathrm{~m}, 2 \mathrm{H}, 19-\mathrm{H}_{\mathrm{b}}, 21-\mathrm{H}_{\mathrm{b}}\right), 1.15(\mathrm{~s}, 3 \mathrm{H}$, 27-H), 1.06-0.99 (m, 1H, 15- $\left.\mathrm{H}_{\mathrm{b}}\right), 1.02(\mathrm{~s}, 3 \mathrm{H}, 23-\mathrm{H}), 0.97(\mathrm{~s}, 3 \mathrm{H}, 25-\mathrm{H}), 0.95-0.87\left(\mathrm{~m}, 1 \mathrm{H}, 1-\mathrm{H}_{\mathrm{b}}\right), 0.90$ $(\mathrm{s}, 6 \mathrm{H}, 29-\mathrm{H}, 30-\mathrm{H}), 0.86-0.82(\mathrm{~m}, 1 \mathrm{H}, 5-\mathrm{H}), 0.82(\mathrm{~s}, 3 \mathrm{H}, 24-\mathrm{H}), 0.78(\mathrm{~s}, 3 \mathrm{H}, 26-\mathrm{H}) \mathrm{ppm} ;{ }^{13} \mathrm{C}-\mathrm{NMR}(101$ $\left.\mathrm{MHz}, \mathrm{CDCl}_{3}\right): \delta=178.3(\mathrm{C}-28), 144.6(\mathrm{C}-13), 122.8$ (C-12), $84.0(\mathrm{C}-3), 67.0$ (C-2), 57.8 (C-32), 55.4 (C-5), 47.7 (C-9), 46.8 (C-19), 46.6 (C-1), 46.5 (C-17), 45.3 (C-33, C-33'), 42.4 (C-18), 42.2 (C-14), 39.6 (C-8), 39.3 (C-4), 38.3 (C-10), 36.9 (C-31), 34.3 (C-21), 33.2 (C-30), 32.8 (C-22), 32.6 (C-7), 30.9 (C-20), 28.8 (C-23), 27.5 (C-15), 25.9 (C-27), 23.8 (C-16), 23.7 (C-11), 23.7 (C-29), 18.5 (C-6), 17.1 (C-24), 16.9 (C-26), 16.8 (C-25) ppm; MS (ESI, MeOH): $\mathrm{m} / \mathrm{z}=543\left(100 \%\right.$, $\left.[\mathrm{M}+\mathrm{H}]^{+}\right)$; analysis calcd. for $\mathrm{C}_{34} \mathrm{H}_{58} \mathrm{~N}_{2} \mathrm{O}_{3}$ (542.85): $\mathrm{C}$ 75.23, H 10.77, N 5.16; found: C 74.99, H 10.97, N 5.08.

(3ß)-N-(2-Pyrrolidin-1-ylethyl)-3-acetyloxy-olean-12-en-28-amide (32), Compound 32 was prepared from 7 according to general procedure $\mathrm{B}$ using 1-(2-aminoethyl)-pyrrolidine as amino compound. Column chromatography $\left(\mathrm{SiO}_{2}, \mathrm{CHCl}_{3} / \mathrm{MeOH} 95: 5\right)$ gave 32 (yield: $90 \%$ ); m.p. $175-178{ }^{\circ} \mathrm{C}$ (decomp.), lit.: $258^{\circ} \mathrm{C}$ (decomp.)[29]; $[\alpha]_{\mathrm{D}}=+47.9^{\circ}$ (c 0.315, $\mathrm{CHCl}_{3}$ ), Lit.: $+49.8^{\circ}$ (c $\left.0.350, \mathrm{CHCl}_{3}\right)$ [29]; $\mathrm{R}_{\mathrm{f}}=0.39$ $\left(\mathrm{CHCl}_{3} / \mathrm{MeOH} 9: 1\right) ; \mathrm{IR}(\mathrm{ATR}): v=2944 \mathrm{~m}, 1731 \mathrm{~m}, 1644 \mathrm{w}, 1522 \mathrm{w}, 1365 \mathrm{~m}, 1244 \mathrm{~s}, 1027 \mathrm{~m}, 985 \mathrm{~m}$, $752 \mathrm{~m} \mathrm{~cm}^{-1} ;{ }^{1} \mathrm{H}-\mathrm{NMR}\left(400 \mathrm{MHz}, \mathrm{CDCl}_{3}\right): \delta=6.89(\mathrm{t}, \mathrm{J}=5.6 \mathrm{~Hz}, 1 \mathrm{H}, \mathrm{NH}), 5.41(\mathrm{t}, \mathrm{J}=3.6 \mathrm{~Hz}, 1 \mathrm{H}$, $12-\mathrm{H}), 4.48(\mathrm{dd}, \mathrm{J}=10.3,5.7 \mathrm{~Hz}, 1 \mathrm{H}, 3-\mathrm{H}), 3.90-3.76\left(\mathrm{~m}, 2 \mathrm{H}, 33-\mathrm{H}_{\mathrm{a}}, 33^{\prime}-\mathrm{H}_{\mathrm{a}}\right), 3.73-3.64\left(\mathrm{~m}, 1 \mathrm{H}, 31-\mathrm{H}_{\mathrm{a}}\right)$, 3.51-3.41 (m, $\left.1 \mathrm{H}, 31-\mathrm{H}_{\mathrm{b}}\right), 3.35-3.18(\mathrm{~m}, 2 \mathrm{H}, 32-\mathrm{H}), 2.99-2.86\left(\mathrm{~m}, 2 \mathrm{H}, 33-\mathrm{H}_{\mathrm{b}}, 33^{\prime}-\mathrm{H}_{\mathrm{b}}\right), 2.64(\mathrm{dd}, \mathrm{J}=13.5$, $4.6 \mathrm{~Hz}, 1 \mathrm{H}, 18-\mathrm{H}), 2.22-1.95\left(\mathrm{~m}, 5 \mathrm{H}, 34-\mathrm{H}, 34^{\prime}-\mathrm{H}, 16-\mathrm{H}_{\mathrm{a}}\right), 2.03$ (s, 3H, Ac), 1.94-1.76 (m, 2H, 11- $\mathrm{H}_{\mathrm{a}}$, $\left.11-\mathrm{H}_{\mathrm{b}}\right), 1.70\left(\mathrm{t}, \mathrm{J}=13.5 \mathrm{~Hz}, 1 \mathrm{H}, 19-\mathrm{H}_{\mathrm{a}}\right), 1.65-1.28\left(\mathrm{~m}, 12 \mathrm{H}, 1-\mathrm{H}_{\mathrm{a}}, 2-\mathrm{H}_{\mathrm{a}}, 2-\mathrm{H}_{\mathrm{b}}, 7-\mathrm{H}_{\mathrm{a}}, 7-\mathrm{H}_{\mathrm{b}}, 16-\mathrm{H}_{\mathrm{b}}, 9-\mathrm{H}\right.$, 6- $\left.\mathrm{H}_{\mathrm{a}}, 15-\mathrm{H}_{\mathrm{a}}, 22-\mathrm{H}_{\mathrm{a}}, 6-\mathrm{H}_{\mathrm{b}}, 21-\mathrm{H}_{\mathrm{a}}\right), 1.28-1.15\left(\mathrm{~m}, 3 \mathrm{H}, 22-\mathrm{H}_{\mathrm{b}}, 19-\mathrm{H}_{\mathrm{b}}, 21-\mathrm{H}_{\mathrm{b}}\right), 1.13(\mathrm{~s}, 3 \mathrm{H}, 27-\mathrm{H}), 1.09-0.97$ $\left(\mathrm{m}, 2 \mathrm{H}, 1-\mathrm{H}_{\mathrm{b}}, 15-\mathrm{H}_{\mathrm{b}}\right), 0.92(\mathrm{~s}, 3 \mathrm{H}, 25-\mathrm{H}), 0.91(\mathrm{~s}, 3 \mathrm{H}, 29-\mathrm{H}), 0.89$ (s, 3H, 30-H), 0.85 (s, 3H, 23-H), 0.84 (s, $3 \mathrm{H}, 24-\mathrm{H}), 0.84-0.79(\mathrm{~m}, 1 \mathrm{H}, 5-\mathrm{H}), 0.71(\mathrm{~s}, 3 \mathrm{H}, 26-\mathrm{H}) \mathrm{ppm} ;{ }^{13} \mathrm{C}-\mathrm{NMR}\left(101 \mathrm{MHz}, \mathrm{CDCl}_{3}\right): \delta=179.9(\mathrm{C}-28)$, 171.1 (Ac), 143.9 (C-13), 123.3 (C-12), 81.0 (C-3), 55.3 (C-5), 55.1 (C-32), 54.8 (C-33, C-33'), 47.6 (C-9), 46.5 (C-19), 46.5 (C-17), 41.9 (C-14), 41.5 (C-18), 39.5 (C-8), 38.2 (C-1), 37.8 (C-4), 37.0 (C-10), 36.4 (C-31), 34.2 (C-21), 33.2 (C-30), 32.9 (C-7), 32.5 (C-22), 30.8 (C-20), 28.2 (C-23), 27.5 (C-15), 25.9 (C-27), 23.6 (C-2), 23.6 (C-29), 23.6 (C-11), 23.6 (C-16), 23.4 (C-34, C-34'), 21.4 (Ac), 18.3 (C-6), 17.2 (C-26), 16.8 (C-24), 15.5 (C-25) ppm; MS (ESI, MeOH): $\left.\mathrm{m} / \mathrm{z}=595.5(100 \% \text {, [M + H] }]^{+}\right)$; analysis calcd. for $\mathrm{C}_{38} \mathrm{H}_{62} \mathrm{~N}_{2} \mathrm{O}_{3}$ (594.93): C 76.72, H 10.50, N 4.71; found: C 76.51, H 10.73, N 4.69.

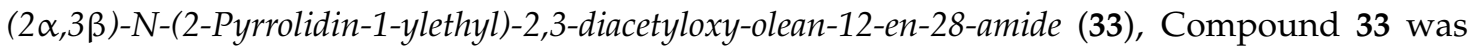
prepared from 8 according to general procedure $\mathrm{B}$ using 1-(2-aminoethyl)-pyrrolidine as amino compound. Column chromatography $\left(\mathrm{SiO}_{2}, \mathrm{CHCl}_{3} / \mathrm{MeOH}\right.$ 9:1) gave 33 (yield: 96\%); m.p. 143-146 ${ }^{\circ} \mathrm{C}$ (decomp.); $[\alpha]_{\mathrm{D}}=+20.0^{\circ}$ (c 0.345, $\left.\mathrm{CHCl}_{3}\right) ; \mathrm{R}_{\mathrm{f}}=0.30\left(\mathrm{CHCl}_{3} / \mathrm{MeOH}\right.$ 95:5); IR (KBr): $v=3426 \mathrm{br} \mathrm{s}, 2948 \mathrm{~s}$, 2802w, 1744s, 1654m, 1508m, 1460m, 1368m, 1250s, 1152w, 1044m cm ${ }^{-1} ;{ }^{1} \mathrm{H}-\mathrm{NMR}\left(400 \mathrm{MHz}, \mathrm{CDCl}_{3}\right)$ : $\delta=6.58(\mathrm{t}, \mathrm{J}=4.3 \mathrm{~Hz}, 1 \mathrm{H}, \mathrm{NH}), 5.30(\mathrm{t}, \mathrm{J}=3.6 \mathrm{~Hz}, 1 \mathrm{H}, 12-\mathrm{H}), 5.08(\mathrm{ddd}, \mathrm{J}=11.5,10.3,4.7 \mathrm{~Hz}, 1 \mathrm{H}, 2-\mathrm{H})$, $4.74(\mathrm{~d}, \mathrm{~J}=10.3 \mathrm{~Hz}, 1 \mathrm{H}, 3-\mathrm{H}), 3.46-3.37\left(\mathrm{~m}, 1 \mathrm{H}, 31-\mathrm{H}_{\mathrm{a}}\right), 3.24-3.13\left(\mathrm{~m}, 1 \mathrm{H}, 31-\mathrm{H}_{\mathrm{b}}\right), 2.67-2.44(\mathrm{~m}, 7 \mathrm{H}$, 32-H, 33-H, 33'-H, 18-H), 2.04 (s, 3H, Ac), 2.03-1.98 (m, 1H, 1- $\left.\mathrm{H}_{\mathrm{a}}\right), 1.97$ (s, 3H, Ac), 1.96-1.83 (m, 3H, $\left.11-\mathrm{H}_{\mathrm{a}}, 11-\mathrm{H}_{\mathrm{b}}, 16-\mathrm{H}_{\mathrm{a}}\right), 1.82-1.76\left(\mathrm{~m}, 4 \mathrm{H}, 34-\mathrm{H}, 34^{\prime}-\mathrm{H}\right), 1.75-1.23\left(\mathrm{~m}, 11 \mathrm{H}, 19-\mathrm{H}_{\mathrm{a}}, 22-\mathrm{H}_{\mathrm{a}}, 16-\mathrm{H}_{\mathrm{b}}, 9-\mathrm{H}\right.$, $\left.22-\mathrm{H}_{\mathrm{b}}, 15-\mathrm{H}_{\mathrm{a}}, 6-\mathrm{H}_{\mathrm{a}}, 7-\mathrm{H}_{\mathrm{a}}, 6-\mathrm{H}_{\mathrm{b}}, 21-\mathrm{H}_{\mathrm{a}}, 7-\mathrm{H}_{\mathrm{b}}\right), 1.24-1.10\left(\mathrm{~m}, 2 \mathrm{H}, 21-\mathrm{H}_{\mathrm{b}}, 19-\mathrm{H}_{\mathrm{b}}\right), 1.14(\mathrm{~s}, 3 \mathrm{H}, 27-\mathrm{H}), 1.05$ $(\mathrm{s}, 3 \mathrm{H}, 25-\mathrm{H}), 1.09-0.99\left(\mathrm{~m}, 2 \mathrm{H}, 1-\mathrm{H}_{\mathrm{b}}, 15-\mathrm{H}_{\mathrm{b}}\right), 0.98-0.93(\mathrm{~m}, 1 \mathrm{H}, 5-\mathrm{H}), 0.90(\mathrm{~s}, 9 \mathrm{H}, 24-\mathrm{H}, 29-\mathrm{H}, 30-\mathrm{H})$, 0.89 (s, 3H, 23-H), 0.77 (s, 3H, 26-H) ppm; ${ }^{13} \mathrm{C}-\mathrm{NMR}\left(101 \mathrm{MHz}, \mathrm{CDCl}_{3}\right): \delta=177.9(\mathrm{C}-28), 170.8$ (Ac), 170.5 (Ac), 144.6 (C-13), 122.3 (C-12), 80.5 (C-3), 70.0 (C-2), 54.8 (C-5), 54.2 (C-32), 53.9 (C-33, C-33'), 47.5 (C-9), 46.7 (C-19), 46.3 (C-17), 43.9 (C-1), 42.3 (C-18), 42.0 (C-14), 39.4 (C-8), 39.3 (C-4), 38.1 (C-10), 37.8 (C-31), 34.2 (C-21), 33.0 (C-30), 32.6 (C-22), 32.3 (C-7), 30.7 (C-20), 28.4 (C-23), 27.3 (C-15), 25.6 (C-27), 23.7 (C-11), 23.7 (C-34, C-34'), 23.6 (C-16), 23.5 (C-29), 21.1 (Ac), 20.9 (Ac), 18.2 (C-6), 17.6 (C-24), 16.8 (C-26), $16.5(\mathrm{C}-25)$ ppm; MS (ESI, MeOH): $\left.\mathrm{m} / \mathrm{z}=653(100 \% \text {, [M + H] }]^{+}\right)$; analysis calcd. for $\mathrm{C}_{40} \mathrm{H}_{64} \mathrm{~N}_{2} \mathrm{O}_{5}$ (652.96): C 73.58, H 9.88, N 4.29; found: C 73.27, H 10.02, N 4.02. 
(3ß)-N-(2-Pyrrolidin-1-ylethyl)-3-hydroxy-olean-12-en-28-amide (37), Compound 37 was prepared from 32 according to general procedure $\mathrm{C}$. Column chromatography $\left(\mathrm{SiO}_{2}, \mathrm{CHCl}_{3} / \mathrm{MeOH} 95: 5\right)$ gave 37 (yield: $76 \%$ ); m.p. $189-192{ }^{\circ} \mathrm{C} ;[\alpha]_{\mathrm{D}}=+40.8^{\circ}$ (c 0.325, MeOH); $\mathrm{R}_{\mathrm{f}}=0.28\left(\mathrm{CHCl}_{3} / \mathrm{MeOH} 9: 1\right) ; \mathrm{IR}$ (ATR): $v=3386 \mathrm{br} w, 2941 \mathrm{~m}, 1631 \mathrm{~m}, 1527 \mathrm{~m}, 1320 \mathrm{~s}, 1031 \mathrm{~m}, 996 \mathrm{~m}, 823 \mathrm{~m}, 752 \mathrm{~m} \mathrm{~cm}^{-1} ;{ }^{1} \mathrm{H}-\mathrm{NMR}(500$ $\left.\mathrm{MHz}, \mathrm{CDCl}_{3}\right): \delta=6.94(\mathrm{t}, \mathrm{J}=5.6 \mathrm{~Hz}, 1 \mathrm{H}, \mathrm{NH}), 5.42(\mathrm{t}, \mathrm{J}=3.7 \mathrm{~Hz}, 1 \mathrm{H}, 12-\mathrm{H}), 3.88-3.78\left(\mathrm{~m}, 2 \mathrm{H}, 33-\mathrm{H}_{\mathrm{a}}\right.$ $\left.33^{\prime}-\mathrm{H}_{\mathrm{a}}\right), 3.73-3.62\left(\mathrm{~m}, 1 \mathrm{H}, 31-\mathrm{H}_{\mathrm{a}}\right), 3.51-3.41\left(\mathrm{~m}, 1 \mathrm{H}, 31-\mathrm{H}_{\mathrm{b}}\right), 3.35-3.22\left(\mathrm{~m}, 2 \mathrm{H}, 33-\mathrm{H}_{\mathrm{b}}, 33^{\prime}-\mathrm{H}_{\mathrm{b}}\right), 3.20$ $(\mathrm{dd}, \mathrm{J}=11.5,4.4 \mathrm{~Hz}, 1 \mathrm{H}, 3-\mathrm{H}), 2.99-2.88(\mathrm{~m}, 2 \mathrm{H}, 32-\mathrm{H}), 2.66(\mathrm{dd}, \mathrm{J}=12.9,3.7 \mathrm{~Hz}, 1 \mathrm{H}, 18-\mathrm{H}), 2.19-2.05$ $\left(\mathrm{m}, 4 \mathrm{H}, 34-\mathrm{H}, 34^{\prime}-\mathrm{H}\right), 2.01\left(\mathrm{ddd}, \mathrm{J}=13.9,13.8,3.9 \mathrm{~Hz}, 1 \mathrm{H}, 16-\mathrm{H}_{\mathrm{a}}\right), 1.97-1.84\left(\mathrm{~m}, 2 \mathrm{H}, 11-\mathrm{H}_{\mathrm{a}}, 11-\mathrm{H}_{\mathrm{b}}\right)$, $1.70\left(\mathrm{t}, \mathrm{J}=13.4 \mathrm{~Hz}, 1 \mathrm{H}, 19-\mathrm{H}_{\mathrm{a}}\right), 1.65-1.29\left(\mathrm{~m}, 12 \mathrm{H}, 1-\mathrm{H}_{\mathrm{a}}, 16-\mathrm{H}_{\mathrm{b}}, 2-\mathrm{H}_{\mathrm{a}}, 2-\mathrm{H}_{\mathrm{b}}, 22-\mathrm{H}_{\mathrm{a}}, 22-\mathrm{H}_{\mathrm{b}}, 9-\mathrm{H}, 6-\mathrm{H}_{\mathrm{a}}\right.$, $\left.15-\mathrm{H}_{\mathrm{a}}, 7-\mathrm{H}_{\mathrm{a}}, 6-\mathrm{H}_{\mathrm{b}}, 21-\mathrm{H}_{\mathrm{a}}\right), 1.28-1.23\left(\mathrm{~m}, 1 \mathrm{H}, 7-\mathrm{H}_{\mathrm{b}}\right), 1.21-1.15\left(\mathrm{~m}, 2 \mathrm{H}, 19-\mathrm{H}_{\mathrm{b}}, 21-\mathrm{H}_{\mathrm{b}}\right), 1.14(\mathrm{~s}, 3 \mathrm{H}, 27-\mathrm{H})$, $1.04\left(\mathrm{ddd}, \mathrm{J}=14.4,3.5,3.4 \mathrm{~Hz}, 1 \mathrm{H}, 15-\mathrm{H}_{\mathrm{b}}\right), 0.98(\mathrm{~s}, 3 \mathrm{H}, 23-\mathrm{H}), 0.97-0.92\left(\mathrm{~m}, 1 \mathrm{H}, 1-\mathrm{H}_{\mathrm{b}}\right), 0.91(\mathrm{~s}, 3 \mathrm{H}$, 29-H), 0.90 (s, 3H, 25-H), 0.89 (s, 3H, 30-H), 0.77 (s, 3H, 24-H), 0.74-0.70 (m, 1H, 5-H), 0.71 (s, 3H, 26-H) ppm; ${ }^{13} \mathrm{C}-\mathrm{NMR}\left(126 \mathrm{MHz}, \mathrm{CDCl}_{3}\right): \delta=180.1$ (C-28), 143.8 (C-13), 123.4 (C-12), 79.1 (C-3), 55.3 (C-5), 55.1 (C-33, C-33'), 54.9 (C-32), 47.7 (C-9), 46.5 (C-17, C-19), 41.9 (C-14), 41.5 (C-18), 39.6 (C-8), 38.9 (C-4), 38.6 (C-1), 37.1 (C-10), 36.4 (C-31), 34.3 (C-21), 33.2 (C-30), 32.9 (C-22), 32.6 (C-7), 30.8 (C-20), 28.2 (C-23), 27.5 (C-15), 27.3 (C-2), 26.0 (C-27), 23.7 (C-29), 23.6 (C-16), 23.5 (C-11), 23.4 (C-34, C-34'), 18.5 (C-6), 17.2 (C-26), 15.7 (C-24), 15.5 (C-25) ppm; MS (ESI, MeOH): $\mathrm{m} / \mathrm{z}=553.5\left(100 \%,[\mathrm{M}+\mathrm{H}]^{+}\right)$; analysis calcd. for $\mathrm{C}_{36} \mathrm{H}_{60} \mathrm{~N}_{2} \mathrm{O}_{2}$ (552.89): C 78.21, $\mathrm{H}$ 10.94, N 5.07; found: $\mathrm{C} 78.01, \mathrm{H} 11.13, \mathrm{~N} 4.83$. (2 $\alpha, 3 \beta)-N$-(2-Pyrrolidin-1-ylethyl)-2,3-dihydroxy-olean-12-en-28-amide (38), Compound 38 was prepared

from 33 according to general procedure $\mathrm{C}$. Column chromatography $\left(\mathrm{SiO}_{2}, \mathrm{CHCl}_{3} / \mathrm{MeOH}\right.$ 9:1) gave 38 (yield: $67 \%$ ); m.p. $153-156^{\circ} \mathrm{C}$ (decomp.); $[\alpha]_{\mathrm{D}}=+44.3^{\circ}$ (c $\left.0.330, \mathrm{CHCl}_{3}\right) ; \mathrm{R}_{\mathrm{f}}=0.10\left(\mathrm{CHCl}_{3} / \mathrm{MeOH}\right.$ 95:5); IR (KBr): $v=3406 \mathrm{br}$ s, 2946s, 2878s, 2808m, 1636s, 1522m, 1462m, 1386m, 1364m, 1268w, 1194w, $1150 \mathrm{w}, 1050 \mathrm{~m} \mathrm{~cm}^{-1},{ }^{1} \mathrm{H}-\mathrm{NMR}\left(400 \mathrm{MHz}, \mathrm{CDCl}_{3}\right): \delta=6.72(\mathrm{t}, \mathrm{J}=5.1 \mathrm{~Hz}, 1 \mathrm{H}, \mathrm{NH}), 5.34(\mathrm{t}, \mathrm{J}=3.6 \mathrm{~Hz}$, $1 \mathrm{H}, 12-\mathrm{H}), 3.67(\mathrm{ddd}, \mathrm{J}=11.3,9.4,4.4 \mathrm{~Hz}, 1 \mathrm{H}, 2-\mathrm{H}), 3.53-3.43\left(\mathrm{~m}, 1 \mathrm{H}, 31-\mathrm{H}_{\mathrm{a}}\right), 3.31-3.22\left(\mathrm{~m}, 1 \mathrm{H}, 31-\mathrm{H}_{\mathrm{b}}\right)$, $2.99(\mathrm{~d}, \mathrm{~J}=9.5 \mathrm{~Hz}, 1 \mathrm{H}, 3-\mathrm{H}), 2.80-2.63\left(\mathrm{~m}, 6 \mathrm{H}, 32-\mathrm{H}, 33-\mathrm{H}, 33^{\prime}-\mathrm{H}\right), 2.56(\mathrm{dd}, \mathrm{J}=13.4,4.3 \mathrm{~Hz}, 1 \mathrm{H}, 18-\mathrm{H})$, 2.02-1.89 (m, 4H, 1- $\left.\mathrm{H}_{\mathrm{a}}, 11-\mathrm{H}_{\mathrm{a}}, 11-\mathrm{H}_{\mathrm{b}}, 16-\mathrm{H}_{\mathrm{a}}\right), 1.89-1.83\left(\mathrm{~m}, 4 \mathrm{H}, 34-\mathrm{H}, 34^{\prime}-\mathrm{H}\right), 1.79-1.24\left(\mathrm{~m}, 11 \mathrm{H}, 19-\mathrm{H}_{\mathrm{a}}\right.$, $\left.22-\mathrm{H}_{\mathrm{a}}, 16-\mathrm{H}_{\mathrm{b}}, 9-\mathrm{H}, 22-\mathrm{H}_{\mathrm{b}}, 6-\mathrm{H}_{\mathrm{a}}, 15-\mathrm{H}_{\mathrm{a}}, 7-\mathrm{H}_{\mathrm{a}}, 6-\mathrm{H}_{\mathrm{b}}, 21-\mathrm{H}_{\mathrm{a}}, 7-\mathrm{H}_{\mathrm{b}}\right), 1.23-1.11\left(\mathrm{~m}, 2 \mathrm{H}, 21-\mathrm{H}_{\mathrm{b}}, 19-\mathrm{H}_{\mathrm{b}}\right), 1.14$ $(\mathrm{s}, 3 \mathrm{H}, 27-\mathrm{H}), 1.08-0.99\left(\mathrm{~m}, 1 \mathrm{H}, 15-\mathrm{H}_{\mathrm{b}}\right), 1.02(\mathrm{~s}, 3 \mathrm{H}, 23-\mathrm{H}), 0.97(\mathrm{~s}, 3 \mathrm{H}, 25-\mathrm{H}), 0.94-0.87\left(\mathrm{~m}, 1 \mathrm{H}, 1-\mathrm{H}_{\mathrm{b}}\right)$, 0.90 (s, 3H, 29-H), 0.89 (s, 3H, 30-H), 0.85-0.79 (m, 1H, 5-H), 0.81 (s, 3H, 24-H), 0.75 (s, 3H, 26-H) ppm; ${ }^{13} \mathrm{C}-\mathrm{NMR}\left(101 \mathrm{MHz}, \mathrm{CDCl}_{3}\right): \delta=178.6$ (C-28), 144.5 (C-13), 122.8 (C-12), 84.0 (C-3), 68.9 (C-2), 55.3 (C-5), 54.6 (C-32), 54.2 (C-33, C-33'), 47.7 (C-9), 46.8 (C-19), 46.5 (C-1), 46.5 (C-17), 42.2 (C-18), 42.1 (C-14), 39.6 (C-8), 39.3 (C-4), 38.3 (C-10), 37.6 (C-31), 34.3 (C-21), 33.2 (C-30), 32.8 (C-22), 32.5 (C-7), 30.8 (C-20), 28.8 (C-23), 27.5 (C-15), 25.9 (C-27), 23.8 (C-11), 23.7 (C-29), 23.7 (C-34, C-34'), 23.6 (C-16), 18.5 (C-6), 17.1 (C-26), 16.9 (C-24), 16.8 (C-25) ppm; MS (ESI, MeOH): m/z = $569\left(100 \%\right.$, [M + H] $\left.{ }^{+}\right)$; analysis calcd. for $\mathrm{C}_{36} \mathrm{H}_{60} \mathrm{~N}_{2} \mathrm{O}_{3}$ (568.89): C 76.01, $\mathrm{H}$ 10.63, N 4.92; found: C 75.86, H 10.90, N 4.77.

(3ß)-N-(2-Piperidin-1-ylethyl)-3-acetyloxy-olean-12-en-28-amide (42), Compound 42 was prepared from 7 according to general procedure B using 1-(2-aminoethyl)-piperidine as amino compound. Column chromatography $\left(\mathrm{SiO}_{2}, \mathrm{CHCl}_{3} / \mathrm{MeOH} 9: 1\right.$ ) gave 42 (yield: $76 \%$ ); m.p. $=163-166^{\circ} \mathrm{C}$ (decomp.); $[\alpha]_{\mathrm{D}}=+47.5^{\circ}\left(\mathrm{c} 0.305, \mathrm{CHCl}_{3}\right) ; \mathrm{R}_{\mathrm{f}}=0.55$ (silica gel, $\mathrm{CHCl}_{3} / \mathrm{MeOH} 9: 1$ ); IR (ATR): $v=2943 \mathrm{~m}, 2864 \mathrm{w}$, $1732 \mathrm{~m}, 1642 \mathrm{w}, 1523 \mathrm{w}, 1432 \mathrm{~m}, 1388 \mathrm{~m}, 1365 \mathrm{~s}, 1330 \mathrm{~m}, 1244 \mathrm{vs}, 1214 \mathrm{~m}, 1149 \mathrm{w}, 1097 \mathrm{w}, 1027 \mathrm{~m}, 1007 \mathrm{~m}$, 986m, 751m; ${ }^{1} \mathrm{H}-\mathrm{NMR}\left(500 \mathrm{MHz}, \mathrm{CDCl}_{3}\right): \delta=6.93(\mathrm{t}, \mathrm{J}=5.3 \mathrm{~Hz}, 1 \mathrm{H}, \mathrm{NH}), 5.42(\mathrm{dd}, \mathrm{J}=3.3 \mathrm{~Hz}, 3.3 \mathrm{~Hz}$, $1 \mathrm{H}, 12-\mathrm{H}), 4.51-4.44(\mathrm{~m}, 1 \mathrm{H}, 3-\mathrm{H}), 3.71\left(\mathrm{dq}, \mathrm{J}=11.9,5.9 \mathrm{~Hz}, 1 \mathrm{H}, 31-\mathrm{H}_{\mathrm{a}}\right), 3.46(\mathrm{dq}, \mathrm{J}=12.2,6.0 \mathrm{~Hz}$, $\left.1 \mathrm{H}, 31-\mathrm{H}_{\mathrm{b}}\right), 3.13(\mathrm{~s}, 2 \mathrm{H}, 32-\mathrm{H}), 2.64(\mathrm{dd}, \mathrm{J}=12.9,3.3 \mathrm{~Hz}, 1 \mathrm{H}, 18-\mathrm{H}), 2.03(\mathrm{~s}, 3 \mathrm{H}, \mathrm{Ac}), 2.02-1.84(\mathrm{~m}, 8 \mathrm{H}$, 2- $\left.\mathrm{H}_{\mathrm{a}}, 2-\mathrm{H}_{\mathrm{b}}, 16-\mathrm{H}_{\mathrm{a}}, 16-\mathrm{H}_{\mathrm{b}} .33-\mathrm{H}, 33^{\prime}-\mathrm{H}\right), 1.70\left(\mathrm{~m}, 1 \mathrm{H}, 19-\mathrm{H}_{\mathrm{a}}\right), 1.66-1.40\left(\mathrm{~m}, 15 \mathrm{H}, 1-\mathrm{H}_{\mathrm{a}}, 11-\mathrm{H}_{\mathrm{a}}, 22-\mathrm{H}_{\mathrm{a}}\right.$, $\left.11-\mathrm{H}_{\mathrm{b}}, 9-\mathrm{H}, 22-\mathrm{H}_{\mathrm{b}}, 6-\mathrm{H}_{\mathrm{a}}, 15-\mathrm{H}_{\mathrm{a}}, 7-\mathrm{H}_{\mathrm{a}}, 34-\mathrm{H}, 34^{\prime}-\mathrm{H}, 35-\mathrm{H}\right), 1.40-1.30\left(\mathrm{~m}, 2 \mathrm{H}, 6-\mathrm{H}_{\mathrm{b}}, 21-\mathrm{H}_{\mathrm{a}}\right), 1.24(\mathrm{~m}$, $\left.1 \mathrm{H}, 7-\mathrm{H}_{\mathrm{b}}\right), 1.22-1.14\left(\mathrm{~m}, 2 \mathrm{H}, 19-\mathrm{H}_{\mathrm{b}}, 21-\mathrm{H}_{\mathrm{b}}\right), 1.13(\mathrm{~s}, 3 \mathrm{H}, 27-\mathrm{H}), 1.04\left(\mathrm{~m}, 2 \mathrm{H}, 1-\mathrm{H}_{\mathrm{b}}, 15-\mathrm{H}_{\mathrm{b}}\right), 0.92(\mathrm{~s}, 3 \mathrm{H}$, 29-H) $0.92(\mathrm{~s}, 3 \mathrm{H}, 26-\mathrm{H}), 0.89(\mathrm{~s}, 3 \mathrm{H}, 30-\mathrm{H}), 0.85(\mathrm{~s}, 3 \mathrm{H}, 23-\mathrm{H}), 0.84(\mathrm{~s}, 3 \mathrm{H}, 25-\mathrm{H}), 0.81(\mathrm{~s}, 1 \mathrm{H}, 5-\mathrm{H}), 0.71$ $(\mathrm{s}, 3 \mathrm{H}, 24-\mathrm{H}) ;{ }^{13} \mathrm{C}-\mathrm{NMR}\left(126 \mathrm{MHz} \mathrm{CDCl}_{3}\right): \delta=179.9$ (C-28), 171.1 (Ac), 143.9 (C-13), 123.3 (C-12), 81.0 (C-3), 56.9 (C-32), 55.3 (C-5), 54.5 (C-33 + C-33'), 47.6 (C-9), 46.5 (C-19), 46.5 (C-17), 41.9 (C-14), 41.5 
(C-18), 39.5 (C-8), 38.2 (C-1), 37.8 (C-4), 37.0 (C-10), 34.9 (C-31), 34.3 (C-21), 33.2 (C-30), 32.9 (C-22), 32.5 (C-7), 30.8 (C-20), 28.2 (C-23), 27.5 (C-15), 26.0 (C-27), 23.6 (C-2), 23.6 (C-29), 23.6 (C-11), 23.6 (C-16), 23.1 (C-34 + C-34'), 22.1 (C-35), 21.4 (Ac), 18.3 (C-6), 17.2 (C-24), 16.8 (C-25), 15.5 (C-26); MS (ESI, MeOH): $\mathrm{m} / \mathrm{z}=609.5\left(100 \%,[\mathrm{M}+\mathrm{H}]^{+}\right)$; analysis calcd. for $\mathrm{C}_{39} \mathrm{H}_{64} \mathrm{~N}_{2} \mathrm{O}_{3}(608.95): \mathrm{C}$ 76.92, $\mathrm{H} 10.59$, N 4.60; found: C 76.78, H 10.79, N 4.44.

$(2 \alpha, 3 \beta)-N$-(2-Piperidin-1-ylethyl)-2,3-diacetyloxy-olean-12-en-28-amide (43), Compound 43 was prepared from 8 according to general procedure $\mathrm{B}$ using 1-(2-aminoethyl)-piperidine as amino compound. Column chromatography $\left(\mathrm{SiO}_{2}, \mathrm{CHCl}_{3} / \mathrm{MeOH} 9: 1\right)$ gave 43 (yield: $71 \%$ ); m.p. $=130-133^{\circ} \mathrm{C}$ (decomp.); $[\alpha]_{\mathrm{D}}=+15.7^{\circ}$ (c $0.35, \mathrm{CHCl}_{3}$ ); $\mathrm{R}_{\mathrm{f}}=0.47$ (silica gel, $\mathrm{CHCl}_{3} / \mathrm{MeOH} 9: 1$ ); IR (ATR): $v=2938 \mathrm{~m}$, 2863w, 1741s, 1653m, 1508m, 1503m, 1466m, 1455m, 1443w, 1434w, 1367m, 1303w, 1247vs, 1229vs, $1155 \mathrm{w}, 1127 \mathrm{w}, 1041 \mathrm{~s}, 1032 \mathrm{~s} ;{ }^{1} \mathrm{H}-\mathrm{NMR}\left(500 \mathrm{MHz}, \mathrm{CDCl}_{3}\right) \delta=6.69(\mathrm{~s}, 1 \mathrm{H}, \mathrm{NH}), 5.36(\mathrm{dd}, \mathrm{J}=3.4 \mathrm{~Hz}, 1 \mathrm{H}$, 12-H), $5.08(\mathrm{td}, \mathrm{J}=11.4,4.6 \mathrm{~Hz}, 1 \mathrm{H}, 2-\mathrm{H}), 4.74(\mathrm{~d}, \mathrm{~J}=10.4 \mathrm{~Hz}, 1 \mathrm{H}, 3-\mathrm{H}), 3.40\left(\mathrm{~m}, 1 \mathrm{H}, 31-\mathrm{H}_{\mathrm{a}}\right), 3.20(\mathrm{~m}, 1 \mathrm{H}$, 31- $\left.\mathrm{H}_{\mathrm{b}}\right), 2.55(\mathrm{~d}, \mathrm{~J}=11.7 \mathrm{~Hz}, 1 \mathrm{H}, 18-\mathrm{H}), 2.43\left(\mathrm{~s}, 6 \mathrm{H}, 32-\mathrm{H}_{\mathrm{a}}, 32-\mathrm{H}_{\mathrm{b}}, 33-\mathrm{H}, 33^{\prime}-\mathrm{H}\right), 2.05$ (s, 3H, Ac), 1.97 (s, 3H, Ac), 2.07-1.82 (m, 3H, 1- $\left.\mathrm{H}_{\mathrm{a}}, 16-\mathrm{H}_{\mathrm{a}}, 16-\mathrm{H}_{\mathrm{b}}\right), 1.78-1.42\left(\mathrm{~m}, 15 \mathrm{H}, 19-\mathrm{H}_{\mathrm{a}}, 11-\mathrm{H}_{\mathrm{a}}, 22-\mathrm{H}_{\mathrm{a}}, 9-\mathrm{H}, 11-\mathrm{H}_{\mathrm{b}}\right.$, 34-H, 34'- $\left.-\mathrm{H}, 6-\mathrm{H}_{\mathrm{a}}, 15-\mathrm{H}_{\mathrm{a}}, 22-\mathrm{H}_{\mathrm{b}}, 35-\mathrm{H}, 7-\mathrm{H}_{\mathrm{a}}\right), 1.42-1.13\left(\mathrm{~m}, 5 \mathrm{H}, 6-\mathrm{H}_{\mathrm{b}}, 21-\mathrm{H}_{\mathrm{a}}, 7-\mathrm{H}_{\mathrm{b}}, 21-\mathrm{H}_{\mathrm{b}}, 19-\mathrm{H}_{\mathrm{b}}\right), 1.14$ (s, 3H, 27-H), $1.04(\mathrm{~s}, 3 \mathrm{H}, 25-\mathrm{H}), 1.09-0.92\left(\mathrm{~m}, 3 \mathrm{H}, 1-\mathrm{H}_{\mathrm{b}}, 15-\mathrm{H}_{\mathrm{b}}, 5-\mathrm{H}\right), 0.90(\mathrm{~s}, 3 \mathrm{H}, 29-\mathrm{H}), 0.90(\mathrm{~s}, 3 \mathrm{H}$, 24-H), 0.90 (s, 3H, 30-H), 0.90 (s, 3H, 23-H), 0.75 (s, 3H, 26-H); ${ }^{13} \mathrm{C}-\mathrm{NMR}\left(126 \mathrm{MHz}, \mathrm{CDCl}_{3}\right) \delta=178.0$, (C-28),170.9 (Ac), 170.7 (Ac), 144.8 (C-13), 122.4 (C-12), 80.7 (C-3), 70.2 (C-2), 57.0 (C-32), 55.0 (C-33 + C-33'), 54.4 (C-5), 47.6 (C-9), 46.9 (C-19), 46.4 (C-17), 44.0 (C-1), 42.3 (C-18), 42.1 (C-14), 39.6 (C-8), 39.5 (C-4), 38.3 (C-10), 35.8 (C-31), 34.3 (C-21), 33.2 (C-30), 32.8 (C-22), 32.3 (C-7), 30.9 (C-20), 28.6 (C-23), 27.4 (C-15), $25.8\left(\mathrm{C}-38+\mathrm{C}-38^{\prime}\right), 25.9(\mathrm{C}-27), 24.2(\mathrm{C}-35), 23.7(\mathrm{C}-16+\mathrm{C}-11), 23.7(\mathrm{C}-29), 21.3(\mathrm{Ac}), 21.0$ (Ac), 18.3 (C-6), 17.8 (C-24), 17.0 (C-26), 16.6 (C-25); MS (ESI, MeOH): m/z = 667.5 ([M + H] $\left.]^{+}, 100 \%\right)$; analysis calcd. for $\mathrm{C}_{41} \mathrm{H}_{66} \mathrm{~N}_{2} \mathrm{O}_{5}$ (666.99): C 73.83, $\mathrm{H}$ 9.97, $\mathrm{N}$ 4.20; found: C 73.69, $\mathrm{H}$ 10.18, $\mathrm{N} 4.01$.

(3ß)-N-(2-Piperidin-1-ylethyl)-3-acetyloxy-20-oxo-30-norlupan-28-amide (45), Compound 45 was prepared from 10 according to general procedure B using 1-(2-aminoethyl)-piperidine as amino compound. Column chromatography $\left(\mathrm{SiO}_{2}, \mathrm{CHCl}_{3} / \mathrm{MeOH}\right.$ 9:1) gave 45 (yield: $63 \%) ;$ m.p. $=141-144{ }^{\circ} \mathrm{C} ;[\alpha]_{\mathrm{D}}=-9.5^{\circ}\left(\mathrm{c} 0.34, \mathrm{CHCl}_{3}\right) ; \mathrm{R}_{\mathrm{f}}=0.43$ (silica gel, $\left.\mathrm{CHCl}_{3} / \mathrm{MeOH}\right) ; \mathrm{IR}(\mathrm{ATR})$ : $v=2939 \mathrm{~m}, 2867 \mathrm{w}, 1733 \mathrm{~m}, 1711 \mathrm{~m}, 1657 \mathrm{~m}, 1654 \mathrm{~m}, 1517 \mathrm{~m}, 1468 \mathrm{~m}, 1451 \mathrm{~m}, 1391 \mathrm{w}, 1367 \mathrm{~m}, 1352 \mathrm{~m}, 1316 \mathrm{w}$, $1302 \mathrm{w}, 1243 \mathrm{vs}, 1196 \mathrm{~m}, 1157 \mathrm{~m}, 1130 \mathrm{w}, 1110 \mathrm{w}, 1090 \mathrm{w}, 1073 \mathrm{w}, 1026 \mathrm{~m}, 979 \mathrm{~m} ;{ }^{1} \mathrm{H}-\mathrm{NMR}\left(500 \mathrm{MHz}, \mathrm{CDCl}_{3}\right)$ $\delta=6.76(\mathrm{~s}, 1 \mathrm{H}, \mathrm{NH}), 4.45(\mathrm{dd}, \mathrm{J}=11.0,5.1 \mathrm{~Hz}, 1 \mathrm{H}, 3-\mathrm{H}), 3.40\left(\mathrm{~m}, 4 \mathrm{H}, 18-\mathrm{H}, 31-\mathrm{H}_{\mathrm{a}}, 31-\mathrm{H}_{\mathrm{b}}\right), 2.63-2.52$ $\left(\mathrm{m}, 6 \mathrm{H}, 32-\mathrm{H}_{\mathrm{a}}, 32-\mathrm{H}_{\mathrm{b}}, 33-\mathrm{H}, 33^{\prime}-\mathrm{H}\right), 2.21-2.14(\mathrm{~m}, 1 \mathrm{H}, 13-\mathrm{H}), 2.15(\mathrm{~s}, 3 \mathrm{H}, 29-\mathrm{H}), 2.09-2.00(\mathrm{~m}, 3 \mathrm{H}, 9-\mathrm{H}$, $\left.16-\mathrm{H}_{\mathrm{a}}, 21-\mathrm{H}_{\mathrm{a}}\right), 2.02(\mathrm{~s}, 3 \mathrm{H}, \mathrm{Ac}), 1.84-1.78\left(\mathrm{dd}, 1 \mathrm{H}, 1-\mathrm{H}_{\mathrm{a}}\right), 1.71-1.53\left(\mathrm{~m}, 8 \mathrm{H}, 34-\mathrm{H}, 34^{\prime}-\mathrm{H}, 19-\mathrm{H}_{\mathrm{a}}, 1-\mathrm{H}_{\mathrm{b}}\right.$, $\left.16-\mathrm{H}_{\mathrm{b}}, 2-\mathrm{H}_{\mathrm{a}}\right), 1.53-1.20\left(\mathrm{~m}, 14 \mathrm{H}, 13-\mathrm{H}_{\mathrm{a}}, 2-\mathrm{H}_{\mathrm{b}}, 21-\mathrm{H}_{\mathrm{b}}, 6-\mathrm{H}_{\mathrm{a}}, 35-\mathrm{H}, 11-\mathrm{H}_{\mathrm{a}}, 12-\mathrm{H}_{\mathrm{a}}, 7-\mathrm{H}_{\mathrm{a}}, 7-\mathrm{H}_{\mathrm{b}}, 6-\mathrm{H}_{\mathrm{b}}, 11-\mathrm{H}_{\mathrm{b}}\right.$, $\left.22-\mathrm{H}_{\mathrm{a}}, 22-\mathrm{H}_{\mathrm{b}}\right), 1.20-1.13\left(\mathrm{~m}, 1 \mathrm{H}, 12-\mathrm{H}_{\mathrm{b}}\right), 1.10-1.00\left(\mathrm{~m}, 2 \mathrm{H}, 15-\mathrm{H}_{\mathrm{a}}, 15-\mathrm{H}_{\mathrm{b}}\right), 0.98(\mathrm{~s}, 3 \mathrm{H}, 27-\mathrm{H}), 0.96-0.91$ (m, 1H, 19- $\left.\mathrm{H}_{\mathrm{b}}\right), 0.88$ (s, 3H, 26-H), $0.82(\mathrm{~s}, 3 \mathrm{H}, 23-\mathrm{H}), 0.82(\mathrm{~s}, 3 \mathrm{H}, 25-\mathrm{H}), 0.81$ (s, 3H, 26-H), 0.79-0.75 $(\mathrm{m}, 1 \mathrm{H}, 5-\mathrm{H}) ;{ }^{13} \mathrm{C}-\mathrm{NMR}\left(126 \mathrm{MHz}, \mathrm{CDCl}_{3}\right) \delta=212.9$ (C-20), 176.5 (Ac), 171.0 (C-28), 81.0 (C-3), 57.3 (C-32), 55.8 (C-5), 55.5 (C-17), 54.3 (C-33), 51.4 (C-18), 50.5 (C-9), 50.1 (C-19), 42.4 (C-14), 40.8 (C-8), 38.5 (C-1), 38.0 (C-4), 37.9 (C-10), 37.2 (C-13), 37.0 (C-22), 35.3 (C-31), 34.4 (C-7), 32.9 (C-16), 30.3 (C-29), 29.6 (C-12), 28.8 (C-21), 28.1 (C-23), 27.3 (C-15), 25.3 (C-34), 23.8 (C-35), 23.8 (C-2), 21.4 (Ac), 21.1 (C-11), 18.3 (C-6), 16.6 (C-24), 16.3 (C-25), 16.3 (C-26), 14.8 (C-27); MS (ESI, MeOH): $\mathrm{m} / \mathrm{z}=611.5\left(100 \%,[\mathrm{M}+\mathrm{H}]^{+}\right)$; analysis calcd. for $\mathrm{C}_{38} \mathrm{H}_{62} \mathrm{~N}_{2} \mathrm{O}_{4}$ (610.92): C 74.71, $\mathrm{H}$ 10.23, $\mathrm{N}$ 4.59; found: $\mathrm{C} 74.56, \mathrm{H}$ 10.51, $\mathrm{N} 4.39$.

(3ß)-N-(2-Piperidin-1-ylethyl)-3-hydroxy-olean-12-en-28-amide (47), Compound 47 was prepared from 42 according to general procedure $\mathrm{C}$. Column chromatography $\left(\mathrm{SiO}_{2}, \mathrm{CHCl}_{3} / \mathrm{MeOH} 95: 5\right)$ gave 47 (yield: 93\%); m.p. $=184-186{ }^{\circ} \mathrm{C}$ (decomp.); $[\alpha]_{\mathrm{D}}=+49.5^{\circ}$ (c $\left.0.365, \mathrm{CHCl}_{3}\right) ; \mathrm{R}_{\mathrm{f}}=0.26$ (silica gel, $\mathrm{CHCl}_{3} / \mathrm{MeOH}$ 9:1); IR (ATR): $v=3372 \mathrm{w}, 2941 \mathrm{~m}, 2864 \mathrm{~m}, 1633 \mathrm{~m}, 1527 \mathrm{~m}, 1431 \mathrm{~s}, 1387 \mathrm{~s}, 1378 \mathrm{~s}, 1357 \mathrm{~s}$, $1324 \mathrm{vs}, 1245 \mathrm{~m}, 1212 \mathrm{~m}, 1200 \mathrm{w}, 1188 \mathrm{w}, 1138 \mathrm{w}, 1093 \mathrm{w}, 1032 \mathrm{~m}, 1006 \mathrm{~m}, 997 \mathrm{~m}, 752 \mathrm{~m} ;{ }^{1} \mathrm{H}-\mathrm{NMR}(500 \mathrm{MHz}$, $\left.\mathrm{CDCl}_{3}\right) \delta=6.97(\mathrm{t}, \mathrm{J}=5.3 \mathrm{~Hz}, 1 \mathrm{H}, \mathrm{NH}), 5.43(\mathrm{dd}, \mathrm{J}=3.4,3.4 \mathrm{~Hz}, 1 \mathrm{H}, 12-\mathrm{H}), 4.58(\mathrm{~s}, 1 \mathrm{H}, \mathrm{OH}), 3.71$ $\left(\mathrm{dq}, \mathrm{J}=11.7,5.8 \mathrm{~Hz}, 1 \mathrm{H}, 31-\mathrm{H}_{\mathrm{a}}\right), 3.46\left(\mathrm{dq}, \mathrm{J}=12.2,5.9 \mathrm{~Hz}, 1 \mathrm{H}, 31-\mathrm{H}_{\mathrm{b}}\right), 3.22-3.17(\mathrm{~m}, 1 \mathrm{H}, 3-\mathrm{H}), 3.17-3.11$ $\left(\mathrm{m}, 2 \mathrm{H}, 32-\mathrm{H}_{\mathrm{a}}, 32-\mathrm{H}_{\mathrm{b}}\right), 2.65(\mathrm{dd}, \mathrm{J}=13.4,3.6 \mathrm{~Hz}, 2 \mathrm{H}, 18-\mathrm{H}), 2.05-1.95\left(\mathrm{~m}, 2 \mathrm{H}, 16-\mathrm{H}_{\mathrm{a}}, 16-\mathrm{H}_{\mathrm{b}}\right), 1.95-1.84$ 
$\left(\mathrm{m}, 8 \mathrm{H}, 11-\mathrm{H}_{\mathrm{a}}, 11-\mathrm{H}_{\mathrm{b}}, 34-\mathrm{H}, 34^{\prime}-\mathrm{H}, 35-\mathrm{H}\right), 1.74-1.67\left(\mathrm{~m}, 1 \mathrm{H}, 19-\mathrm{H}_{\mathrm{a}}\right), 1.65-1.45\left(\mathrm{~m}, 10 \mathrm{H}, 1-\mathrm{H}_{\mathrm{a}}, 16-\mathrm{H}_{\mathrm{a}}\right.$, $\left.22-\mathrm{H}_{\mathrm{a}}, 16-\mathrm{H}_{\mathrm{b}}, 22-\mathrm{H}_{\mathrm{b}}, 2-\mathrm{H}_{\mathrm{a}}, 2-\mathrm{H}_{\mathrm{b}}, 9-\mathrm{H}, 6-\mathrm{H}_{\mathrm{a}}, 15-\mathrm{H}_{\mathrm{a}}\right), 1.45-1.39\left(\mathrm{~m}, 1 \mathrm{H}, 7-\mathrm{H}_{\mathrm{a}}\right), 1.40-1.29\left(\mathrm{~m}, 2 \mathrm{H}, 6-\mathrm{H}_{\mathrm{b}}\right.$ ， 21- $\left.\mathrm{H}_{\mathrm{a}}\right), 1.29-1.22\left(\mathrm{~m}, 1 \mathrm{H}, 7-\mathrm{H}_{\mathrm{b}}\right), 1.21-1.15\left(\mathrm{~m}, 2 \mathrm{H}, 19-\mathrm{H}_{\mathrm{b}}, 21-\mathrm{H}_{\mathrm{b}}\right), 1.14(\mathrm{~s}, 3 \mathrm{H}, 27-\mathrm{H}), 1.07-1.00(\mathrm{~m}, 1 \mathrm{H}$, $\left.15-\mathrm{H}_{\mathrm{b}}\right), 0.97(\mathrm{~s}, 3 \mathrm{H}, 23-\mathrm{H}), 0.97-0.93\left(\mathrm{~m}, 1 \mathrm{H}, 1-\mathrm{H}_{\mathrm{b}}\right), 0.92(\mathrm{~s}, 3 \mathrm{H}, 29-\mathrm{H}), 0.89(\mathrm{~s}, 3 \mathrm{H}, 25-\mathrm{H}), 0.89(\mathrm{~s}, 3 \mathrm{H}$, 30-H), 0.77 (s, 3H, 24-H), $0.73(\mathrm{~s}, 1 \mathrm{H}, 5-\mathrm{H}), 0.71(\mathrm{~s}, 3 \mathrm{H}, 26-\mathrm{H}) ;{ }^{13} \mathrm{C}-\mathrm{NMR}\left(126 \mathrm{MHz}, \mathrm{CDCl}_{3}\right) \delta=179.9$ (C-28), 143.8 (C-13), 123.4 (C-12), 79.1 (C-3), 55.3 (C-32), 56.9 (C-5), 54.5 (C-33 + C-33'), 47.7 (C-9), 46.5 (C-19), 46.5 (C-17), 41.9 (C-14), 41.5 (C-18), 39.5 (C-8), 38.9 (C-4), 38.6 (C-1), 37.1 (C-10), 34.9 (C-31), 34.3 (C-21), 33.2 (C-30), 32.9 (C-22), 32.6 (C-7), 30.8 (C-20), 28.2 (C-23), 27.5 (C-15), 27.3 (C-2), 26.0 (C-27), 23.6 (C-29), 23.6 (C-16), 23.6 (C-11), 23.0 (C-34 + C-34'), 22.1 (C-35), 18.4 (C-6), 17.2 (C-26), 15.7 (C-24), $15.5(\mathrm{C}-25)$; $\mathrm{MS}(\mathrm{ESI}, \mathrm{MeOH}): \mathrm{m} / \mathrm{z}=567.5\left(100 \%\right.$, $\left.[\mathrm{M}+\mathrm{H}]^{+}\right)$; analysis calcd. for $\mathrm{C}_{37} \mathrm{H}_{62} \mathrm{~N}_{2} \mathrm{O}_{2}(566.92)$ : C 78.39, H 11.02, N 4.94; found: C 78.21,H 11.18, N 4.78.

$(2 \alpha, 3 \beta)-N$-(2-Piperidin-1-ylethyl)-2,3-dihydroxy-olean-12-en-28-amide (48), Compound 48 was prepared from 43 according to general procedure $\mathrm{C}$. Column chromatography $\left(\mathrm{SiO}_{2}, \mathrm{CHCl}_{3} / \mathrm{MeOH}\right.$ 9:1) gave 48 (yield: $87 \%$ ); m.p. $=149-152{ }^{\circ} \mathrm{C} ;[\alpha]_{\mathrm{D}}=+45.2^{\circ}\left(\mathrm{c} 0.31, \mathrm{CHCl}_{3}\right) ; \mathrm{R}_{\mathrm{f}}=0.21$ (silica gel, $\mathrm{CHCl}_{3} / \mathrm{MeOH}$ 9:1); IR (ATR): $v=3378 \mathrm{~m}, 2934 \mathrm{vs}, 2861 \mathrm{~m}, 2853 \mathrm{~m}, 1635 \mathrm{~s}, 1513 \mathrm{~s}, 1459 \mathrm{~s}, 1454 \mathrm{~s}, 1386 \mathrm{~s}$, 1378s, 1364m, 1348m, 1328w, 1303m, 1281m, 1266m, 1259m, 1232w, 1209w, 1194m, 1154m, 1129m, $1111 \mathrm{w}, 1096 \mathrm{~m}, 1084 \mathrm{~m}, 1050 \mathrm{vs}, 1016 \mathrm{~m}, 992 \mathrm{~m}, 958 \mathrm{~m}, 660 \mathrm{~m} ;{ }^{1} \mathrm{H}-\mathrm{NMR}\left(500 \mathrm{MHz}, \mathrm{CDCl}_{3}\right) \delta=5.41(\mathrm{~s}, 1 \mathrm{H}$, 12-H), 3.74-3.64 (m, 1H, 2-H), 3.59-3.45 (m, 1H, 31- $\left.\mathrm{H}_{\mathrm{a}}\right), 3.43-3.25\left(\mathrm{~m}, 1 \mathrm{H}, 31-\mathrm{H}_{\mathrm{b}}\right), 3.00(\mathrm{~d}, \mathrm{~J}=9.5 \mathrm{~Hz}, 1 \mathrm{H}$, $3-\mathrm{H}), 2.72-2.60(\mathrm{~m}, 1 \mathrm{H}, 18-\mathrm{H}), 2.64-2.36\left(\mathrm{~m}, 2 \mathrm{H}, 32-\mathrm{H}_{\mathrm{a}}, 32-\mathrm{H}_{\mathrm{b}}\right), 2.02-1.92\left(\mathrm{~m}, 4 \mathrm{H}, 1-\mathrm{H}_{\mathrm{a}}, 16-\mathrm{H}_{\mathrm{a}}, 16-\mathrm{H}_{\mathrm{b}}\right.$, 11- $\left.\mathrm{H}_{\mathrm{a}}\right), 1.77-1.49\left(\mathrm{~m}, 17 \mathrm{H}, 1-\mathrm{H}_{\mathrm{b}}, 33-\mathrm{H}, 33^{\prime}-\mathrm{H}, 9-\mathrm{H}, 11-\mathrm{H}_{\mathrm{b}}, 34-\mathrm{H}, 34^{\prime}-\mathrm{H}, 6-\mathrm{H}_{\mathrm{a}}, 22-\mathrm{H}_{\mathrm{a}}, 35-\mathrm{H}, 7-\mathrm{H}_{\mathrm{a}}, 15-\mathrm{H}_{\mathrm{a}}\right)$, $1.50-1.42\left(\mathrm{~m}, 1 \mathrm{H}, 7-\mathrm{H}_{\mathrm{b}}\right), 1.41-1.31\left(\mathrm{~m}, 2 \mathrm{H}, 6-\mathrm{H}_{\mathrm{b}}, 21-\mathrm{H}_{\mathrm{a}}\right), 1.31-1.28\left(\mathrm{~m}, 1 \mathrm{H}, 21-\mathrm{H}_{\mathrm{b}}\right), 1.19\left(\mathrm{~m}, 3 \mathrm{H}, 22-\mathrm{H}_{\mathrm{b}}\right.$, 19- $\left.\mathrm{H}_{\mathrm{a}}\right), 1.15(\mathrm{~s}, 3 \mathrm{H}, 27-\mathrm{H}), 1.04\left(\mathrm{~m}, 1 \mathrm{H}, 15-\mathrm{H}_{\mathrm{b}}\right), 1.03(\mathrm{~s}, 3 \mathrm{H}, 23-\mathrm{H}), 0.97(\mathrm{~s}, 3 \mathrm{H}, 25-\mathrm{H}), 0.93(\mathrm{~s}, 3 \mathrm{H}, 29-\mathrm{H})$, $0.91(\mathrm{~s}, 3 \mathrm{H}, 30-\mathrm{H}), 0.92-0.88\left(\mathrm{~m}, 1 \mathrm{H}, 19-\mathrm{H}_{\mathrm{b}}\right), 0.86-0.82(\mathrm{~m}, 1 \mathrm{H}, 5-\mathrm{H}), 0.82(\mathrm{~s}, 3 \mathrm{H}, 26-\mathrm{H}), 0.74(\mathrm{~s}, 3 \mathrm{H}, 24-\mathrm{H})$; ${ }^{13} \mathrm{C}-\mathrm{NMR}(126 \mathrm{MHz}, \mathrm{CDCl}$ ) $\delta=179.0$ (C-28), 144.0 (C-13), 122.8 (C-12), 84.1 (C-3), 69.1 (C-2), 57.2 (C-32), 55.37 (C-33 + C-33'), 54.5 (C-5), 48.2 (C-17), 47.7 (C-9), 46.5 (C-19), 46.5 (C-1), 43.2 (C-18), 42.1 (C-14), 39.6 (C-8), 39.3 (C-4), 39.1 (C-10), 38.4 (C-31), 34.3 (C-21), 33.2 (C-30), 33.0 (C-22), 32.5 (C-7), 30.9 (C-20), 28.8 (C-23), 27.5 (C-15), 25.8 (C-34 + C-34'), 26.0 (C-27), 24.1 (C-35), 23.7 (C-16), 23.7 (C-11), 23.7 (C-29), 18.5 (C-6), 17.2 (C-24), 16.9 (C-26), 16.8 (C-25); MS (ESI, MeOH): m/z = $569.5\left([\mathrm{M}+\mathrm{H}]^{+}, 100 \%\right)$; analysis calcd. for $\mathrm{C}_{37} \mathrm{H}_{62} \mathrm{~N}_{2} \mathrm{O}_{3}$ (582.91): C 76.24, $\mathrm{H}$ 10.72, $\mathrm{N}$ 4.81; found: C 75.97, H 10.93, N 4.57.

(3ß)-N-(2-Piperidin-1-ylethyl)-3-hydroxy-20-oxo-30-norlupan-28-amide (50), Compound $\mathbf{5 0}$ was prepared from 45 according to general procedure $\mathrm{C}$. Column chromatography $\left(\mathrm{SiO}_{2}, \mathrm{CHCl}_{3} / \mathrm{MeOH}\right.$ 9:1) gave 50 (yield: $86 \%$ ); m.p. $=153-156{ }^{\circ} \mathrm{C}$ (decomp.); $[\alpha]_{\mathrm{D}}=-21.2^{\circ}\left(\mathrm{c} 0.115, \mathrm{CHCl}_{3}\right) ; \mathrm{R}_{\mathrm{f}}=0.16$ (silica gel, $\mathrm{CHCl}_{3} / \mathrm{MeOH}$ ); IR (ATR): $v=3371 \mathrm{w}, 2934 \mathrm{vs}, 2865 \mathrm{~m}, 1706 \mathrm{~m}, 1643 \mathrm{~s}, 1524 \mathrm{~s}, 1519 \mathrm{~s}, 1466 \mathrm{~s}, 1450 \mathrm{~s}$, $1387 \mathrm{~m}, 1376 \mathrm{~s}, 1356 \mathrm{~s}, 1328 \mathrm{~m}, 1319 \mathrm{~m}, 1301 \mathrm{~m}, 1277 \mathrm{~m}, 1245 \mathrm{~s}, 1197 \mathrm{~s}, 1161 \mathrm{~m}, 1131 \mathrm{~m}, 1109 \mathrm{~m}, 1085 \mathrm{~m}, 1046 \mathrm{~s}$, 1035s, 1003m, 987m, 973m; ${ }^{1} \mathrm{H}-\mathrm{NMR}\left(500 \mathrm{MHz}, \mathrm{CDCl}_{3}\right) \delta=3.67-3.55\left(\mathrm{~m}, 2 \mathrm{H}, 31-\mathrm{H}_{\mathrm{a}}, 31-\mathrm{H}_{\mathrm{b}}\right), 3.27(\mathrm{td}$, $\left.\mathrm{J}=11.2,3.9 \mathrm{~Hz}, 2 \mathrm{H}, 1-\mathrm{H}_{\mathrm{a}}, 1-\mathrm{H}_{\mathrm{b}}\right), 3.17-3.07\left(\mathrm{~m}, 3 \mathrm{H}, 32-\mathrm{H}_{\mathrm{a}}, 32-\mathrm{H}_{\mathrm{b}}, 3-\mathrm{H}\right), 2.20-2.12(\mathrm{~m}, 1 \mathrm{H}, 13-\mathrm{H}), 2.09$ (s, 3H, 28-H), 2.10-2.02 (m, 1H, 16- $\left.\mathrm{H}_{\mathrm{a}}\right), 2.00-1.94(\mathrm{~m}, 1 \mathrm{H}, 18-\mathrm{H}), 1.91-1.73\left(\mathrm{~m}, 6 \mathrm{H}, 12-\mathrm{H}_{\mathrm{a}}, 34-\mathrm{H}, 34^{\prime}-\mathrm{H}\right.$, $\left.22-\mathrm{H}_{\mathrm{a}}\right), 1.62-1.06\left(\mathrm{~m}, 22 \mathrm{H}, 19-\mathrm{H}_{\mathrm{a}}, 33-\mathrm{H}, 33^{\prime}-\mathrm{H}, 15-\mathrm{H}_{\mathrm{a}}, 15-\mathrm{H}_{\mathrm{b}}, 2-\mathrm{H}_{\mathrm{a}}, 16-\mathrm{H}_{\mathrm{b}}, 6-\mathrm{H}_{\mathrm{a}}, 22-\mathrm{H}_{\mathrm{b}}, 12-\mathrm{H}_{\mathrm{b}}, 11-\mathrm{H}_{\mathrm{a}}\right.$, $\left.6-\mathrm{H}_{\mathrm{b}}, 7-\mathrm{H}_{\mathrm{a}}, 35-\mathrm{H}, 7-\mathrm{H}_{\mathrm{b}}, 21-\mathrm{H}_{\mathrm{a}}, 9-\mathrm{H}, 11-\mathrm{H}_{\mathrm{b}}, 21-\mathrm{H}_{\mathrm{b}}\right), 1.05-0.92\left(\mathrm{~m}, 1 \mathrm{H}, 2-\mathrm{H}_{\mathrm{b}}\right), 0.90(\mathrm{~s}, 3 \mathrm{H}, 27-\mathrm{H}), 0.89$ $(\mathrm{s}, 3 \mathrm{H}, 23-\mathrm{H}), 0.82(\mathrm{~s}, 3 \mathrm{H}, 25-\mathrm{H}), 0.85-0.78\left(\mathrm{~m}, 1 \mathrm{H}, 19-\mathrm{H}_{\mathrm{b}}\right), 0.74(\mathrm{~s}, 3 \mathrm{H}, 24-\mathrm{H}), 0.68(\mathrm{~s}, 3 \mathrm{H}, 26-\mathrm{H}), 0.63-0.58$ $(\mathrm{m}, 1 \mathrm{H}, 5-\mathrm{H}) ;{ }^{13} \mathrm{C}-\mathrm{NMR}\left(126 \mathrm{MHz}, \mathrm{CDCl}_{3}\right) \delta=212.7$ (C-20), 177.8 (C-30), 78.7 (C-3), 56.9 (C-32), 55.6 (C-17), 55.3 (C-5), 54.0 (C-33), 51.1 (C-19), 50.4 (C-9), 50.0 (C-18), 42.1 (C-14), 40.6 (C-8), 38.8 (C-4), 38.6 (C-1), 37.6 (C-22), 37.1 (C-10), 36.7 (C-13), 34.3 (C-7), 34.2 (C-31), 32.1 (C-16), 30.0 (C-28), 29.4 (C-21), 28.4 (C-12), 28.0 (C-23), 27.3 (C-15), 27.2 (C-2), 22.8 (C-34), 21.8 (C-35), 20.90 (C-11), 18.2 (C-6), 16.1 (C-24), 16.1 (C-25), 15.4 (C-26), 14.6 (C-27); MS (ESI, MeOH): $\left.\mathrm{m} / \mathrm{z}=568.5(100 \% \text {, [M + H] }]^{+}\right)$; analysis calcd. for $\mathrm{C}_{36} \mathrm{H}_{60} \mathrm{~N}_{2} \mathrm{O}_{3}$ (568.89): C 76.01, $\mathrm{H}$ 10.63, N 4.92; found: C 75.93, H 10.46, N 4.71. 


\section{Conclusions}

In this study, 40 triterpenoic acid amides (11-50), with different triterpenoic backbones and various amine residues were synthesized and subjected to Ellman's assay to determine their potential as inhibitors of cholinesterases. Furthermore, some enzyme kinetic studies were performed. Thus, systematic variation of the amine substituent led to analogs possessing the same or even better BChE-inhibiting properties as standard galantamine hydrobromide. Outstanding derivatives were 2-pyrrolidin-1-ylethyl substituted compounds 34 (from BA), 36 (from UA), and 37 (from OA), showing $\mathrm{K}_{\mathrm{i}}$ values of $0.39 \pm 0.04 \mu \mathrm{M}, 0.47 \pm 0.08 \mu \mathrm{M}$, and $0.55 \pm 0.02 \mu \mathrm{M}$, respectively. Furthermore, Ellman's assay revealed several platanic acid compounds as excellent inhibitors of BChE. Particularly, 40, 45, and $\mathbf{5 0}$ were great inhibitors, showing inhibition rates even in the nanomolar range. The most active compound in the test was a hybrid holding a platanic acid backbone and a pyrrolidinyl residue. For (3ß)-N-(2-pyrrolidin-1-ylethyl)-3-acetyloxy-20-oxo-30- norlupan-28-amide (35), inhibition constants $\mathrm{K}_{\mathrm{i}}=0.07 \pm 0.01 \mu \mathrm{M}$ and $\mathrm{K}_{\mathrm{i}}{ }^{\prime}=2.38 \pm 0.48 \mu \mathrm{M}$ have been determined. The results obtained in the biological assay can be explained by appropriate molecular modeling calculations. All active compounds were mixed-type BChE inhibitors with a dominating competitive part $\left(\mathrm{K}_{\mathrm{i}}<\mathrm{K}_{\mathrm{i}}{ }^{\prime}\right)$. The best inhibitor for acetylcholinesterase was a betulinic acid derived piperidinyl derivative (49), acting as a mixed-type inhibitor showing $\mathrm{K}_{\mathrm{i}}=1.00 \pm 0.09 \mu \mathrm{M}$ and $\mathrm{K}_{\mathrm{i}}{ }^{\prime}=1.42 \pm 0.08 \mu \mathrm{M}$, respectively.

Supplementary Materials: Supplementary data related to this article can be found online.

Author Contributions: A.L., M.K., and R.C., conceived and designed the experiments; M.K. and I.S. performed the experiments; A.L., performed the biological assays and experiments; W.B., performed the molecular modeling; A.L., M.K., and R.C., analyzed the data and wrote the paper.

Funding: We acknowledge the financial support within the funding program Open Access Publishing by the German Research Foundation (DFG).

Acknowledgments: We'd like to thank R. Kluge for measuring the ESI-MS spectra and D. Ströhl and his team for the NMR spectra. Thanks are also due to J. Wiese and V. Simon for the IR spectra and optical rotations.

Conflicts of Interest: The authors declare no conflict of interest.

\section{References}

1. Alzheimer, A. Über eine eigenartige Erkrankung der Hirnrinde. Allg. Z. Psychiatr. Psych.-Gerichtl. Med. 1907, 64, 146-148.

2. Davies, P. Selective loss of central cholinergic neurons in Alzheimer's disease. Lancet 1976, 308, 1403. [CrossRef]

3. Peron, G.; Marzaro, G.; Dall'Acqua, S. Known Triterpenes and their Derivatives as Scaffolds for the Development of New Therapeutic Agents for Cancer. Curr. Med. Chem. 2018, 25, 1259-1269. [CrossRef] [PubMed]

4. Rezanka, T.; Siristova, L.; Sigler, K. Sterols and Triterpenoids with Antiviral Activity. Anti-Infect. Agents Med. Chem. 2009, 8, 193-210. [CrossRef]

5. Xiao, S.; Tian, Z.; Wang, Y.; Si, L.; Zhang, L.; Zhou, D. Recent progress in the antiviral activity and mechanism study of pentacyclic triterpenoids and their derivatives. Med. Res. Rev. 2018, 38, 951-976. [CrossRef] [PubMed]

6. Cunha, L.C.S.; e Silva, M.L.A.; Furtado, N.A.J.C.; Vinhólis, A.H.C.; Martins, C.H.G.; da Silva Filho, A.; Cunha, W.R. Antibacterial Activity of Triterpene Acids and Semi-Synthetic Derivatives against Oral Pathogens. Z. Naturforsch. C 2007, 62, 668-672. [CrossRef]

7. Wolska, K.; Grudniak, A.; Fiecek, B.; Kraczkiewicz-Dowjat, A.; Kurek, A. Antibacterial activity of oleanolic and ursolic acids and their derivatives. Open Life Sci. 2010, 5, 543-553. [CrossRef]

8. Song, Q.-Y.; Qi, W.-Y.; Li, Z.-M.; Zhao, J.; Chen, J.-J.; Gao, K. Antifungal activities of triterpenoids from the roots of Astilbe myriantha Diels. Food Chem. 2011, 128, 495-499. [CrossRef] [PubMed]

9. Jamila, N.; Khairuddean, M.; Yeong, K.K.; Osman, H.; Murugaiyah, V. Cholinesterase inhibitory triterpenoids from the bark of Garcinia hombroniana. J. Enzyme Inhib. Med. Chem. 2015, 30, 133-139. [CrossRef] [PubMed]

10. Ahmad, Z.; Mehmood, S.; Ifzal, R.; Malik, A.; Afza, N.; Ashraf, M.; Jahan, E. A New Ursane-type Triterpenoid from Salvia santolinifolia. Turk. J. Chem. 2007, 31, 495-501. 
11. Schwarz, S.; Loesche, A.; Lucas, S.D.; Sommerwerk, S.; Serbian, I.; Siewert, B.; Pianowski, E.; Csuk, R. Converting maslinic acid into an effective inhibitor of acylcholinesterases. Eur. J. Med. Chem. 2015, 103, 438-445. [CrossRef] [PubMed]

12. Heller, L.; Kahnt, M.; Loesche, A.; Grabandt, P.; Schwarz, S.; Brandt, W.; Csuk, R. Amino derivatives of platanic acid act as selective and potent inhibitors of butyrylcholinesterase. Eur. J. Med. Chem. 2017, 126, 652-668. [CrossRef] [PubMed]

13. Loesche, A.; Köwitsch, A.; Lucas, S.D.; Al-Halabi, Z.; Sippl, W.; Al-Harrasi, A.; Csuk, R. Ursolic and oleanolic acid derivatives with cholinesterase inhibiting potential. Bioorganic Chem. 2019, 85, 23-32. [CrossRef] [PubMed]

14. Wiemann, J.; Loesche, A.; Csuk, R. Novel dehydroabietylamine derivatives as potent inhibitors of acetylcholinesterase. Bioorg. Chem. 2017, 74, 145-157. [CrossRef] [PubMed]

15. Loesche, A.; Wiemann, J.; Rohmer, M.; Brandt, W.; Csuk, R. Novel 12-Hydroxydehydroabietylamine derivatives act as potent and selective butyrylcholinesterase inhibitors. Bioorg. Chem.. submitted.

16. Perry, E.K.; Perry, R.H.; Blessed, G.; Tomlinson, B.E. Changes in brain cholinesterases in senile dementia of Alzheimer type. Neuropathol. Appl. Neurobiol. 1978, 4, 273-277. [CrossRef] [PubMed]

17. Dixon, M. The determination of enzyme inhibitor constants. Biochem. J. 1953, 55, 170-171. [CrossRef] [PubMed]

18. Cornish-Bowden, A. A simple graphical method for determining the inhibition constants of mixed, uncompetitive and non-competitive inhibitors. Biochem. J. 1974, 137, 143-144. [CrossRef] [PubMed]

19. Lineweaver, H.; Burk, D. The Determination of Enzyme Dissociation Constants. J. Am. Chem. Soc. 1934, 56, $658-666$. [CrossRef]

20. Sommerwerk, S.; Csuk, R. Convenient and chromatography-free partial syntheses of maslinic acid and augustic acid. Tetrahedron Lett. 2014, 55, 5156-5158. [CrossRef]

21. Sommerwerk, S.; Heller, L.; Serbian, I.; Csuk, R. Straightforward partial synthesis of four diastereomeric 2,3-dihydroxy-olean-12-en-28-oic acids from oleanolic acid. Tetrahedron 2015, 71, 8528-8534. [CrossRef]

22. Kahnt, M.; Heller, L.; Grabandt, P.; Al-Harrasi, A.; Csuk, R. Platanic acid: A new scaffold for the synthesis of cytotoxic agents. Eur. J. Med. Chem. 2018, 143, 259-265. [CrossRef] [PubMed]

23. Kahnt, M.; Al-Harrasi, A.; Csuk, R. Ethylenediamine Derived Carboxamides of Betulinic and Ursolic Acid as Potential Cytotoxic Agents. Molecules 2018, 23, 2558. [CrossRef] [PubMed]

24. Deng, S.-L.; Baglin, I.; Nour, M.; Cavé, C. Synthesis of phosphonodipeptide conjugates of ursolic acid and their homologs. Heteroat. Chem. 2008, 19, 55-65. [CrossRef]

25. Wong, M.H.L.; Bryan, H.K.; Copple, I.M.; Jenkins, R.E.; Chiu, P.H.; Bibby, J.; Berry, N.G.; Kitteringham, N.R.; Goldring, C.E.; O’Neill, P.M.; et al. Design and Synthesis of Irreversible Analogues of Bardoxolone Methyl for the Identification of Pharmacologically Relevant Targets and Interaction Sites. J. Med. Chem. 2016, 59, 2396-2409. [CrossRef] [PubMed]

26. Siewert, B.; Pianowski, E.; Obernauer, A.; Csuk, R. Towards cytotoxic and selective derivatives of maslinic acid. Bioorg. Med. Chem. 2014, 22, 594-615. [CrossRef] [PubMed]

27. Petrenko, N.I.; Elantseva, N.V.; Petukhova, V.Z.; Shakirov, M.M.; Shul'ts, E.E.; Tolstikov, G.A. Synthesis of betulonic acid derivatives containing amino-acid fragments. Chem. Nat. Compd. 2002, 38, 331-339. [CrossRef]

28. Vystrčil, A.; Buděšínský, M. Triterpenes. XVI. Unusual epimerisation of the C(19)-acetyl group of 20-oxo-30-norlupane derivatives. Collect. Czech. Chem. Commun. 1970, 35, 295-311. [CrossRef]

29. Heller, L.; Knorrscheidt, A.; Flemming, F.; Wiemann, J.; Sommerwerk, S.; Pavel, I.Z.; Al-Harrasi, A.; Csuk, R. Synthesis and proapoptotic activity of oleanolic acid derived amides. Bioorg. Chem. 2016, 68, 137-151. [CrossRef] [PubMed]

Sample Availability: Samples of all compounds are available from the authors. 